\title{
A Keplerian disk around a Class 0 source: ALMA observations of VLA1623A
}

\author{
Nadia M. Murillo ${ }^{1}$, Shih-Ping Lai ${ }^{2,3}$, Simon Bruderer ${ }^{1}$, Daniel Harsono ${ }^{4,5}$, and Ewine F. van Dishoeck ${ }^{1,4}$ \\ ${ }^{1}$ Max-Planck-Institut für extraterrestrische Physik, Giessenbachstraße 1, 85748 Garching bei München, Germany \\ e-mail: nmurillo@mpe.mpg.de \\ 2 Institute of Astronomy and Department of Physics, National Tsing Hua University, 101 Section 2 Kuang Fu Road, 30013 Hsinchu, \\ Taiwan \\ 3 Academia Sinica Institute of Astronomy and Astrophysics, PO Box 23-141, 10617 Taipei, Taiwan \\ ${ }^{4}$ Leiden Observatory, Leiden University, Niels Bohrweg 2, 2300 RA Leiden, The Netherlands \\ 5 SRON Netherlands Institute for Space Research, PO Box 800, 9700 AV Groningen, The Netherlands
}

Received 26 August 2013 / Accepted 29 October 2013

\section{ABSTRACT}

Context. Rotationally supported disks are critical in the star formation process. The questions of when they form and what factors influence or hinder their formation have been studied but are largely unanswered. Observations of early-stage YSOs are needed to probe disk formation.

Aims. VLA1623 is a triple non-coeval protostellar system, with a weak magnetic field perpendicular to the outflow, whose Class 0 component, VLA1623A, shows a disk-like structure in continuum with signatures of rotation in line emission. We aim to determine whether this structure is in part or in whole a rotationally supported disk, i.e. a Keplerian disk, and what its characteristics are.

Methods. ALMA Cycle 0 Early Science $1.3 \mathrm{~mm}$ continuum and $\mathrm{C}^{18} \mathrm{O}(2-1)$ observations in the extended configuration are presented here and used to perform an analysis of the disk-like structure using position-velocity (PV) diagrams and thin disk modeling with the addition of foreground absorption.

Results. The PV diagrams of the $\mathrm{C}^{18} \mathrm{O}$ line emission suggest the presence of a rotationally supported component with a radius of at least $50 \mathrm{AU}$. Kinematical modeling of the line emission shows that the disk out to $180 \mathrm{AU}$ is actually rotationally supported, with the rotation described well by Keplerian rotation out to at least $150 \mathrm{AU}$, and the central source mass is $\sim 0.2 M_{\odot}$ for an inclination of $55^{\circ}$. Pure infall and conserved angular momentum rotation models are excluded.

Conclusions. VLA1623A, a very young Class 0 source, presents a disk with an outer radius $R_{\text {out }}=180 \mathrm{AU}$ with a Keplerian velocity structure out to at least $150 \mathrm{AU}$. The weak magnetic fields and recent fragmentation in this region of $\rho$ Ophiuchus may have played a leading role in the formation of the disk.

Key words. stars: formation - ISM: individual objects: VLA1623 - accretion, accretion disks - methods: observational stars: low-mass - techniques: interferometric

\section{Introduction}

Disks are key actors in the formation of stars. They are crucial for accretion and angular momentum distribution in the early stages and for planet formation in the later stages. Rotationally supported disks have been observed using molecular lines in the Class II stage of star formation (Mannings \& Sargent 1997; Guilloteau \& Dutrey 1998; Guilloteau et al. 1999; Qi et al. 2004; Hughes et al. 2009; Rodriguez et al. 2010), while continuum disk-like structures, the so-called pseudo-disks, are reported in Class 0 objects (Jørgensen et al. 2009; Enoch et al. $2009,2011)$. This leads to the expectation that rotationally supported or Keplerian disks evolve from pseudo-disks between the Class 0 and II stages.

Idealized, non-magnetized conditions for a collapsing isothermal core suggest that a small rotating disk $\left(R_{\text {out }}<\right.$ $100 \mathrm{AU}$ ) should form as early as the Class 0 stage and grow as $R \propto t^{3}$, where $t$ is time since collapse (Terebey et al. 1984). Adding magnetic fields to the problem offers varied results. Ideal MHD shows that magnetic field breaking can hinder disk formation (Mellon \& Li 2008); however, if the magnetic

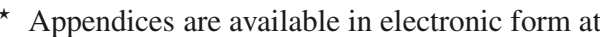
http: //www . aanda.org
}

field and rotation axes are misaligned, rotationally supported disks may form since the magnetic breaking efficiency is reduced (Hennebelle \& Ciardi 2009; Krumholz et al. 2013). Consideration of non-ideal MHD effects and their role in disk formation has been explored, but there is no clear solution ( $\mathrm{Li}$ et al. 2011).

Despite the observational relations and predictions obtained from simulations, it is still unclear when disks actually begin to form and to what degree factors, such as magnetic fields and fragmentation, hinder or encourage the formation of rotationally supported disks.

While observations of (sub-)Keplerian disks in Class I YSOs (Hogerheijde 2001; Brinch et al. 2007; Lommen et al. 2008; Jørgensen et al. 2009; Takakuwa et al. 2012; Yen et al. 2013) support the expectation that disks form between the Class 0 and II stages, recent observations have found indications that rotationally supported disks may be present as early as the Class 0 stage (NGC 1333 IRAS4A: Choi et al. 2010; L1527: Tobin et al. 2012; VLA1623: Murillo \& Lai 2013). Interestingly, hourglass-like magnetic fields are present in NGC 1333 IRAS4A (Girart et al. 2006; Hull et al. 2013b) and in the large scale in L1527 (Davidson et al. 2011), while magnetic fields perpendicular to the outflow direction are present in VLA1623 
Table 1. VLA1623's 1.3 mm continuum fluxes measured with ALMA.

\begin{tabular}{lcccc}
\hline \hline Source & RA & Dec & Peak $\left(\mathrm{mJy} \mathrm{beam}^{-1}\right)$ & Integrated $(\mathrm{mJy})$ \\
\hline VLA1623A & $16: 26: 26.390$ & $-24: 24: 30.688$ & 93.1 & $201.3 \pm 1.2$ \\
VLA1623B & $16: 26: 26.309$ & $-24: 24: 30.588$ & 93.5 & $96.3 \pm 0.7$ \\
VLA1623W & $16: 26: 25.636$ & $-24: 24: 29.488$ & 21.6 & $37.3 \pm 0.9$ \\
\hline
\end{tabular}

(Holland et al. 1996; Hull et al. 2013a,b) and in the small scale in L1527 (Hull et al. 2013b). Additionally, while NGC 1333 IRAS4A and VLA1623 are confirmed multiples, it is unclear whether L1527 is a protobinary (Loinard et al. 2002) or a single protostar. This brings back the question of what factors play a role in the formation of a Keplerian disk and if the combination of these factors is more relevant than the evolutionary stage of a protostar when it comes to the formation of a disk.

VLA1623 is a triple non-coeval protostellar system (Murillo \& Lai 2013; Chen et al. 2013) with a prominent outflow (André et al. 1990) located in $\rho$ Ophiuchus $(d \sim 120 \mathrm{pc}$, Loinard et al. 2008). VLA1623 is composed of three continuum sources: VLA1623A, a deeply embedded Class 0 source with no emission shortward of $24 \mu \mathrm{m}$; VLA1623B, a very cold and compact source separated 1.1" from VLA1623A; and VLA1623W, a Class I source at a projected distance of $10^{\prime \prime}$ from VLA1623A. SMA observations of VLA1623 revealed that the easternmost component, VLA1623A, has a disk-like structure in $\mathrm{C}^{18} \mathrm{O}$ and $\mathrm{C}^{17} \mathrm{O}$ that was mimicked in continuum. Furthermore, VLA1623A's disk shows a velocity gradient characteristic of rotation. A simple eyeball analysis of the position-velocity diagrams of the $\mathrm{C}^{17} \mathrm{O}$ and $\mathrm{C}^{18} \mathrm{O}$ emission by Murillo \& Lai (2013) suggested that the disk was rotationally supported, most likely exhibiting pure Keplerian rotation. They concluded that further analysis is required to distinguish the pseudo-disk $v \propto R^{-1}$ pattern from the rotationally supported $v \propto R^{-0.5}$ profile.

In this paper, we present Atacama Large Millimeter/submillimeter Array (ALMA) Early Science Cycle 0 observations of VLA1623A in $1.3 \mathrm{~mm}$ continuum and $\mathrm{C}^{18} \mathrm{O}$ emission. The results of the $\mathrm{C}^{18} \mathrm{O}$ ALMA observations are consistent with previous Sub-Millimeter Array (SMA) $\mathrm{C}^{18} \mathrm{O}$ observations in that they both show the disk-like structure and velocity gradient centered on VLA1623A. However, the sensitivity and spatial and velocity resolutions of the detection are significantly increased and improved due to ALMA's capabilities. To determine whether the $\mathrm{C}^{18} \mathrm{O}$ emission is tracing a rotationally supported disk about VLA1623A, we performed position-velocity diagram analysis and modeled the $\mathrm{C}^{18} \mathrm{O}$ emission in order to determine the extent to which the observed structure is rotationally supported.

\section{Observations}

We observed VLA1623 (pointing coordinates $\alpha=16: 26: 26.419$, $\delta=-24.24 .29 .988$, J2000) with the Atacama Large Millimeter/submillimeter Array (ALMA) during the early science Cycle 0 period on April 8, 2012. Observations were done in Band 6 (230 GHz) using the extended configuration, consisting of 16 antennae with a maximum baseline of $\sim 400 \mathrm{~m}$, for a total observing time of one hour and a duty cycle of $58 \%$. Calibration was done with 3C 279, 1733-130, and Titan for bandpass, gain, and flux calibration, respectively. The spectral configuration was set up to observe four molecular lines: $\mathrm{DCO}^{+}(3-2), \mathrm{C}^{18} \mathrm{O}(2-1)$, $\mathrm{N}_{2} \mathrm{D}^{+}(3-2)$, and ${ }^{12} \mathrm{CO}(2-1)$, in addition to continuum. No significant $\mathrm{N}_{2} \mathrm{D}^{+}$emission was detected. In this paper we only analyze and discuss the results of $\mathrm{C}^{18} \mathrm{O}$ and continuum. The remaining lines will be discussed in future papers. The spectral configuration provided a velocity resolution of $0.0833 \mathrm{~km} \mathrm{~s}^{-1}$ for $\mathrm{C}^{18} \mathrm{O}$.

Reduced data were received on June 15, 2012. We redid the data calibration and reduction using the standard pipeline for single-pointing observations. Comparison of the delivered data and our recalibrated data shows consistency in the continuum flux levels. The synthesized beam size is $0.79^{\prime \prime} \times 0.54^{\prime \prime}$ for the continuum and $0.79^{\prime \prime} \times 0.61^{\prime \prime}$ for $\mathrm{C}^{18} \mathrm{O}$, providing enough resolution to resolve the continuum and line emission from each source.

We compare the $1.3 \mathrm{~mm}$ continuum observations obtained with ALMA (this work) and SMA (Murillo \& Lai 2013). To compare, VLA1623B's integrated flux is used since it was the most sensitive to the flux calibrations. We use the same region and task (CASA's imstat task) to measure the integrated flux for both datasets. For the SMA data, VLA1623B has an integrated flux of $96.8 \pm 0.7 \mathrm{mJy}$, while for the ALMA data, VLA1623B has an integrated flux of $96.3 \pm 0.7 \mathrm{mJy}$. This shows that both observations are consistent.

James Clark Webb Telescope (JCMT) observations towards VLA1623 by Jørgensen et al. (2002) detected $\mathrm{C}^{18} \mathrm{O}(2-1)$ with a flux of $12.1 \mathrm{~K} \mathrm{~km} \mathrm{~s}^{-1}$ (174 $\mathrm{Jy} \mathrm{km} \mathrm{s}^{-1}$, with $\left.14.46 \mathrm{Jy} / \mathrm{K}\right)$. Our $\mathrm{C}^{18} \mathrm{O}(2-1)$ detection with ALMA has a flux of $13 \mathrm{Jy} \mathrm{km} \mathrm{s}{ }^{-1}$. Thus, we recover $\sim 8 \%$ of the total single-dish detected flux.

\section{Results}

\section{1. $1.3 \mathrm{~mm}$ continuum}

ALMA $1.3 \mathrm{~mm}$ continuum observations detect three continuum sources (Fig. 1, top left), in agreement with previous SMA observations (Murillo \& Lai 2013; Chen et al. 2013). The easternmost source, VLA1623A, shows an elongated and flattened morphology peaking in the center, with the elongation perpendicular to the outflow direction (Fig. 1). VLA1623A is detected at $24 \mu \mathrm{m}$, but shows no emission shortward of $24 \mu \mathrm{m}$ (Fig. 1, bottom row). VLA1623B, located to the west of VLA1623A and separated by $1.1^{\prime \prime}$, is compact and shows no discernible infrared emission (Fig. 1, bottom row) suggesting that it is deeply embedded and cold. The westernmost source, VLA1623W ( 10" separation), is observed to be compact and dimmer than the other two sources in millimeter, but is bright in the infrared (Fig. 1, bottom row). Although VLA1623W is located at the edge of the field of view in our ALMA observations, it is a $\sim 20 \sigma$ detection, and its flux and position coincide with previous detections of this source, so we consider it a genuine detection. Peak and integrated fluxes of each source are listed in Table 1. Integrated fluxes for each source were obtained by integrating the continuum emission over a region the size of the source out to $3 \sigma$. In this paper, we focus on VLA1623A, whose SED is shown in the top right of Fig. 1.

\section{2. $\mathrm{C}^{18} \mathrm{O}(2-1)$}

The $\mathrm{C}^{18} \mathrm{O}(2-1)$ emission towards VLA1623A shows an elongated and flattened structure perpendicular to the outflow 

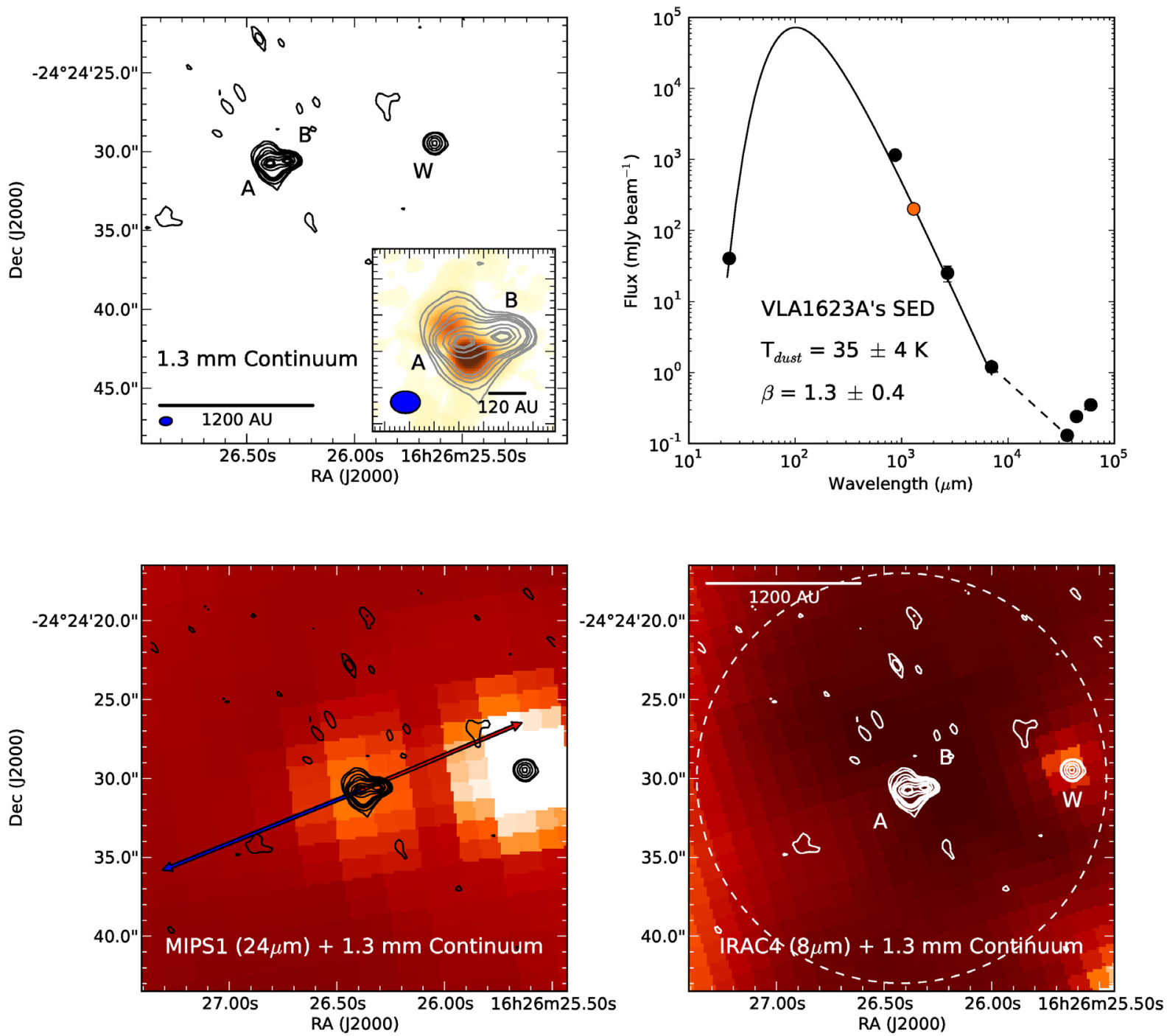

Fig. 1. VLA1623 in continuum. Top left: ALMA $1.3 \mathrm{~mm}$ continnum, three components are observed: VLA1623A, B, and W. While B and W are observed to be compact, A shows an extended and flattened component. The inset shows a zoom-in of VLA1623A and B (black contours), overlaid with intensity-integrated $\mathrm{C}^{18} \mathrm{O}(2-1)$ (halftone). Contours are in steps of $3 \sigma, 5 \sigma, 10 \sigma, 15 \sigma, 20 \sigma, 40 \sigma, 60 \sigma$, and $78 \sigma$, with $\sigma=1 \mathrm{mJy}^{2}$ beam ${ }^{-1}$. Top right: VLA1623A's SED; the orange point indicates VLA1623A's integrated continuum flux obtained from our ALMA observations. The solid line is the best fit of a single-temperature graybody fit, and the resulting parameters are indicated in the figure. Flux uncertainties are usually smaller than the plot symbols. Bottom left: ALMA $1.3 \mathrm{~mm}$ continuum contours overlaid on Spitzer MIPS1. Contours are the same as in the top left panel. Red and blue arrows indicate the red- and blue-shifted large-scale outflow direction. Bottom right: ALMA 1.3 mm continuum contours overlaid on Spitzer IRAC4. Contours are the same as in the top left panel. The dashed circle indicates the field of view of the ALMA observations.

direction, following the same shape as VLA1623A's $1.3 \mathrm{~mm}$ continuum emission (Fig. 1, top left insert, Fig. 2, center). Results and discussion of the $\mathrm{C}^{18} \mathrm{O}$ emission towards VLA1623B and $\mathrm{W}$ are presented in Appendix A.

The $\mathrm{C}^{18} \mathrm{O}$ emission about VLA1623A exhibits a velocity gradient along the major axis, with blueshifted material to the $\mathrm{NE}$ and redshifted material to the SW in the velocity range of 2 to $6.5 \mathrm{~km} \mathrm{~s}^{-1}$ (Fig. 2). The results of our ALMA observations presented here are consistent with SMA observations of $\mathrm{C}^{18} \mathrm{O}(2-1)$ toward VLA1623A (Murillo \& Lai 2013). In comparison, however, our ALMA observations have higher sensitivity (rms noise $\left.=18 \mathrm{mJy}_{\text {beam }}^{-1}\right)$ and velocity resolution $\left(0.0833 \mathrm{~km} \mathrm{~s}^{-1}\right)$ than previous SMA observations $\left(\mathrm{rms}\right.$ noise $=42 \mathrm{mJy} \mathrm{beam}^{-1}$ and velocity resolution $=0.275 \mathrm{~km} \mathrm{~s}^{-1}$ ). ALMA's higher sensitivity and velocity resolution allow us to obtain a better view of the structure being traced and a deeper kinematical analysis. In addition, the high sensitivity allowed detecting previously unknown filament-like features, located to the north and south of
VLA1623A's $\mathrm{C}^{18} \mathrm{O}$ disk structure (Fig. 2, center). These features may be the beginnings of a disk wind (Klaassen et al. 2013).

The observed velocity gradient along the major axis suggests rotation (Fig. 2 left and center), as previously suspected from the SMA detection of $\mathrm{C}^{18} \mathrm{O}$. Furthermore, lack of a velocity gradient along the outflow axis indicates that the observed $\mathrm{C}^{18} \mathrm{O}$ line emission is tracing only the envelope and/or disk structure (Fig. 2 right).

The $\rho$ Ophiuchus region is known to have several layers of foreground clouds along the line of sight (Loren 1989), as shown by studies toward cores B and E in $\rho$ Ophiuchus (Loren 1989; Boogert et al. 2002; van Kempen et al. 2009). However, there are no current studies of the foreground of VLA1623 $(\rho$ Ophiuchus core A). From JCMT observations toward VLA1623, it is difficult to determine the position of absorbing material given the dense and active region (Jørgensen et al. 2004). Determining the foreground over a large area from single-dish data requires a more in-depth study beyond the scope of this paper. Thus to 

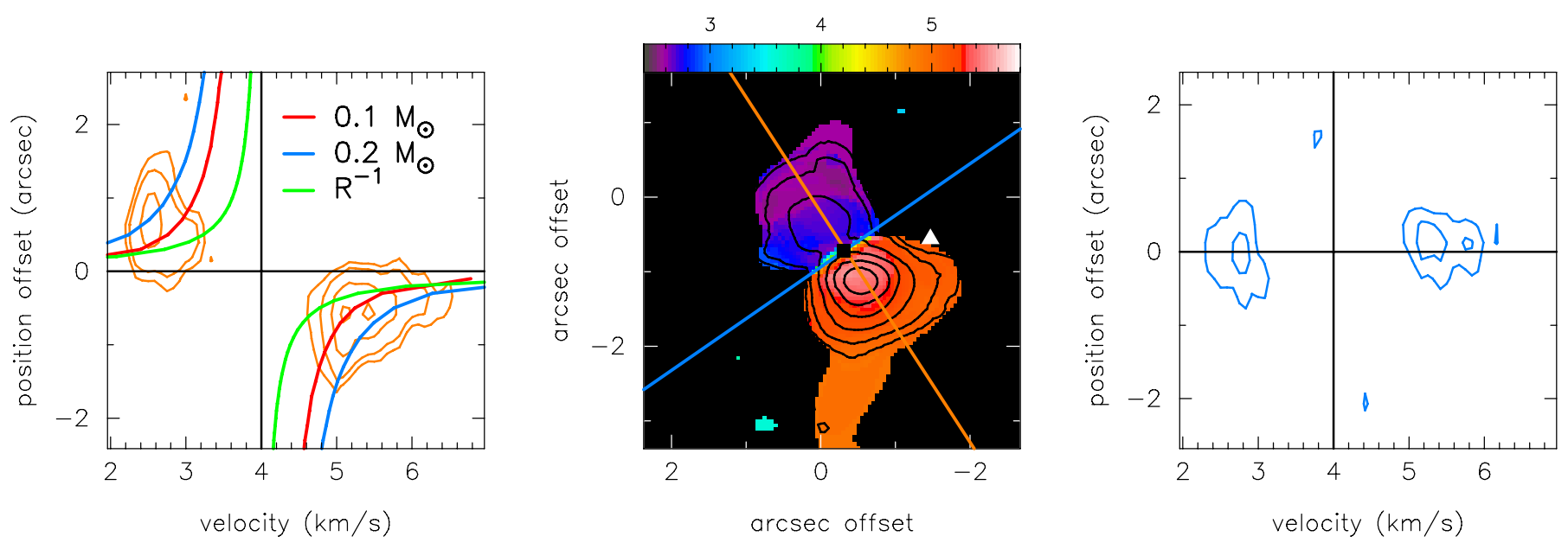

Fig. 2. Observed $\mathrm{C}^{18} \mathrm{O}(2-1)$. Left: pure Keplerian rotation curves $\left(v \propto R^{-0.5}\right.$, red and blue) and infall $\left(v \propto R^{-1}\right.$, green) are overlaid on the PV diagram. This suggests that the emission may be rotationally supported with $M_{*} \sim 0.1-0.2 M_{\odot}$. Center: $\mathrm{C}^{18} \mathrm{O}$ velocity map (moment 1 , halftone) and intensity integrated (moment 0 , contours) maps. Contours are in steps of $3 \sigma, 5 \sigma, 10 \sigma, 15 \sigma, 20 \sigma$ and $25 \sigma$ with $\sigma=13$ mJy beam $^{-1}$. VLA1623A and B's positions are marked with a square and triangle, respectively. Orange and blue lines indicate the image-space PV diagram cuts at $\mathrm{PA}=35^{\circ}$ (left) and $125^{\circ}$ (right), respectively. Right: lack of velocity gradient suggests no outflow contamination on the $\mathrm{C}^{18} \mathrm{O}$ emission. In both PV diagrams contours are in steps of $3 \sigma, 5 \sigma, 10 \sigma$ and $15 \sigma$ where $\sigma=19$ mJy beam $^{-1}$ and the black lines indicate the systemic velocity and position of VLA1623A.

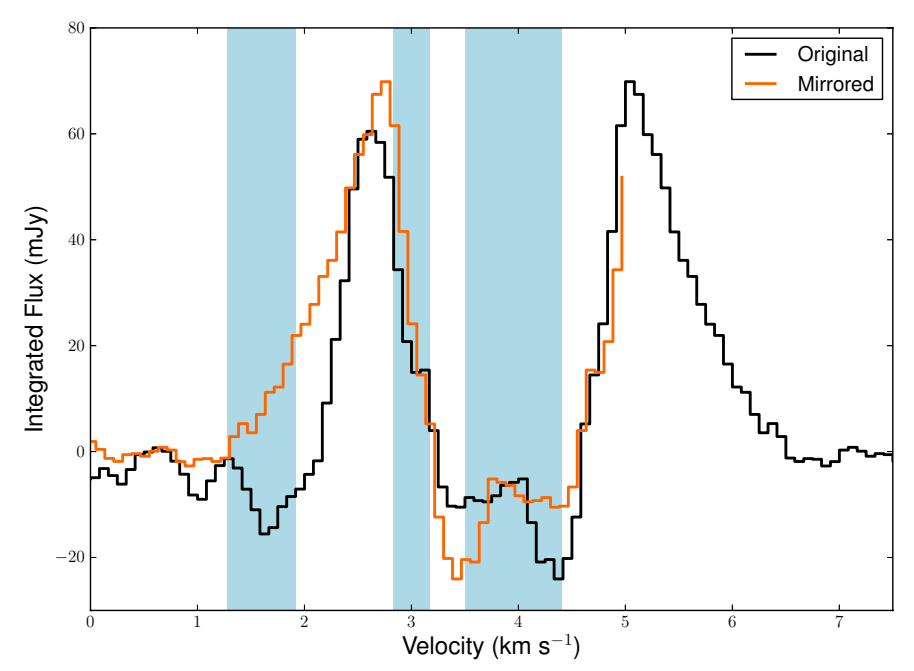

Fig. 3. $\mathrm{C}^{18} \mathrm{O}(2-1)$ line profile. The unaltered spectral profile (black) is overlaid with a portion of the spectral profile mirrored about $4.0 \mathrm{~km} \mathrm{~s}^{-1}$ (orange). Light blue areas mark the widths obtained from the best- fit thin disk model (Table 3 ) of the foreground material $\left(1.6\right.$ and $\left.3 \mathrm{~km} \mathrm{~s}^{-1}\right)$ and the envelope $\left(4.0 \mathrm{~km} \mathrm{~s}^{-1}\right)$. The horizontal line between 5 and $6.5 \mathrm{~km} \mathrm{~s}^{-1}$ shows the velocity range used for the UV-space PV diagram.

estimate the positions of the absorbing material, we compared the original and mirrored spectra (Fig. 3). From line observations, Mardones et al. (1997) and Yu \& Chernin (1997) report VLA1623's systemic velocity $v_{\mathrm{lsr}}$ to be between 3.4 and $3.8 \mathrm{~km} \mathrm{~s}^{-1}$. Mirroring the spectra about this range of $v_{\mathrm{lsr}}$ does not produce a symmetric spectra, however. We instead find that the $\mathrm{C}^{18} \mathrm{O}$ spectra is better mirrored about $4.0 \mathrm{~km} \mathrm{~s}^{-1}$, which would suggest that VLA1623's $v_{\text {lsr }}$ might be $4.0 \mathrm{~km} \mathrm{~s}^{-1}$. We then consider the full range of $v_{\mathrm{lsr}}=3.4$ to $4.0 \mathrm{~km} \mathrm{~s}^{-1}$ in this work. Regarding the absorbing material, it is clear from Fig. 3 that there is absorption at velocities near 2 and $3 \mathrm{~km} \mathrm{~s}^{-1}$, as well as absorption towards the systemic velocity. The absorption at the systemic velocity is either due to absorption caused by VLA1623's outer envelope or to resolved-out emission. On the other hand, the absorptions near 2 and $3 \mathrm{~km} \mathrm{~s}^{-1}$ are more likely to be due to foreground clouds. These foreground clouds "eat out" some of the blueshifted emission, causing the observed red- and blueshifted $\mathrm{C}^{18} \mathrm{O}$ emission to seem asymmetric towards VLA1623A.

\section{Analysis}

\subsection{Position-velocity diagrams}

The first and most common way of analyzing the kinematics of line emission is through position-velocity (PV) diagrams. Here we perform image-space and UV-space PV diagrams. Imagespace PV diagrams project the line-emission 3D data cube into a 2D plane along a cut, defined by position angle (PA) and source position, over the structure of interest. UV-space PV diagrams, on the other hand, are constructed by fitting, channel by channel, the UV visibilities of the line emission to find the peak position in each channel (Lommen et al. 2008; Jørgensen et al. 2009). The peak positions of each channel are then rotated to the PA along the velocity gradient, projected onto PV space and fit with a power law in log-log space. The resulting PV diagram and power law fit provide insight into the kinematic structure of the line emission.

The $\mathrm{C}^{18} \mathrm{O}$ velocity integrated map (Fig. 2, center) shows a velocity gradient along the major axis $\left(\mathrm{PA}=35 \pm 15^{\circ}\right)$ of the structure, perpendicular to the outflow. The image-space PV diagram along the structure's major axis (Fig. 2, left) is characteristic of a rotating structure around a 0.1 to $0.2 M_{\odot}$ object, with pure infall clearly providing a worse fit. The rotation curves in Fig. 2 (left panel) do not account for inclination effects.

Figure 4 shows the UV-space PV diagram of the $\mathrm{C}^{18} \mathrm{O}$ emission. The blueshifted emission is excluded from the diagram since it is strongly affected by foreground emission (Fig. 3, also see Sect. 3.2) at higher velocities. Thus we only fit the redshifted points, with velocities greater than $5.0 \mathrm{~km} \mathrm{~s}^{-1}$. The velocity range of points used is shown in Fig. 3. The data points are fit with a power law of the form $v=a R^{n}$, and we find that the $\mathrm{UV}$-space PV diagram is independent of the inclination angle of 


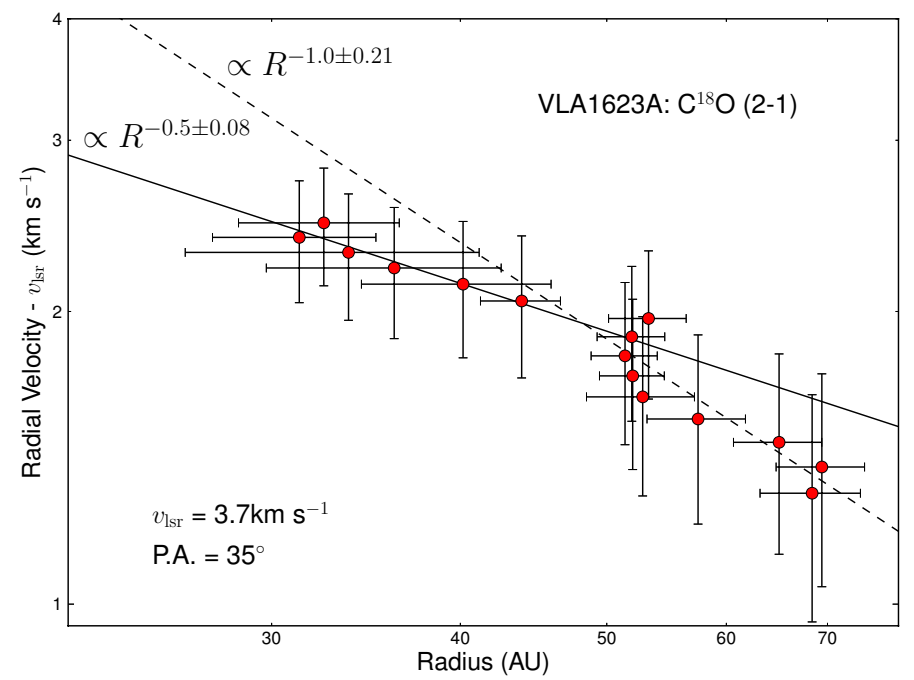

Fig. 4. UV-space PV diagram of $\mathrm{C}^{18} \mathrm{O} . v_{\mathrm{lsr}}=3.7 \mathrm{~km} \mathrm{~s}^{-1}$. The red points indicate the redshifted $\left(v>5.0 \mathrm{~km} \mathrm{~s}^{-1}\right)$ emission. The velocity range covered in this diagram is indicated in Fig. 3. Blueshifted emission $(v<$ $2.8 \mathrm{~km} \mathrm{~s}^{-1}$ ) is not included in the diagram since it is strongly affected by foreground emission (see Fig. 3 and Sect. 3.2). The points are fit with a power law of the form $v=a R^{n}$. The solid line shows the fit to the highvelocity points $\left(v>5.7 \mathrm{~km} \mathrm{~s}^{-1}\right)$, while the dashed line shows the fit to the low-velocity points $\left(v<5.7 \mathrm{~km} \mathrm{~s}^{-1}\right)$. This suggests that the pure Keplerian disk extends to a radius of $50 \mathrm{AU}$.

the structure. The entire range of possible $v_{\mathrm{lsr}}\left(3.4\right.$ to $\left.4.0 \mathrm{~km} \mathrm{~s}^{-1}\right)$ was fit. The results of the fit for any $v_{\mathrm{lsr}}$ are within the error of the fit presented here, which uses the mean $v_{\mathrm{lsr}}, 3.7 \mathrm{~km} \mathrm{~s}^{-1}$. Thus we find that the high-velocity points $\left(v>5.7 \mathrm{~km} \mathrm{~s}^{-1}\right)$ are well fit by $v \propto R^{-0.5 \pm 0.08}$, giving a central protostellar mass $M_{*}$ of $0.22 \pm 0.02 M_{\odot}$. On the other hand, the low-velocity points $\left(5 \mathrm{~km} \mathrm{~s}^{-1}<v<5.7 \mathrm{~km} \mathrm{~s}^{-1}\right)$ are well fit by $v \propto R^{-1}$. This suggests that there is a turnover point at $50 \mathrm{AU}$, where the inner parts, i.e. $R \leq 50 \mathrm{AU}$, are under the effect of pure Keplerian rotation and the outer parts are undergoing infall. In the following section, we argue that the lower velocity points are affected by optical depth and absorption, and that $R$ can be larger.

\subsection{Modeling of $\mathrm{C}^{18} \mathrm{O}(2-1)$}

Based on the results from the PV diagrams, which suggest there is a rotationally supported component in the observed $\mathrm{C}^{18} \mathrm{O}$ emission, we proceed to further investigate the structure and its kinematics using an analytical thin disk model with the addition of absorbing foreground material. The model does not include radiative transfer or excitation since the goal is to study the kinematics and structure of the observed disk. The input parameters include the outer radius $R_{\text {out }}$ of the disk, source position, PA, inclination $i$ of the disk, the mass of the central source $M_{*}$, and the temperature and column density gradients of the disk surface. Generated maps are convolved to the observed clean beam. The model output is in the form of position-positionvelocity (xyv) data cubes in FITS format.

For all of the models we fix the central protostellar mass $M_{*}=0.2 M_{\odot}$, which was obtained from the PV diagram analysis (see Sect. 4.1 ), and the position angle to $35^{\circ}$, since these parameters are well constrained from the PV diagrams (see Sect. 4.1 and Figs. 2 and 4). We set $v_{\text {lsr }}=4.0 \mathrm{~km} \mathrm{~s}^{-1}$, which is the symmetry axis of the spectrum. The distance is set to $120 \mathrm{pc}$, and the source position is set slightly offset by $0.02^{\prime \prime} \times 0.04^{\prime \prime}$ to the SW from
VLA1623A's position to match the center of the $\mathrm{C}^{18} \mathrm{O}$ emission, i.e. the rotation axis.

Other free parameters in the model are line width $v_{\text {width }}$, inclination $i$, and outer radius $R_{\text {out }}$. These values are constrained to best fit the observations. The attempted value ranges for each parameter are $0.1 \mathrm{~km} \mathrm{~s}^{-1} \leq v_{\text {width }} \leq 1.0 \mathrm{~km} \mathrm{~s}^{-1} ; 0^{\circ}$ (face-on) $\leq$ $i \leq 90^{\circ}$ (edge-on); $100 \mathrm{AU} \leq R_{\text {out }} \leq 200 \mathrm{AU}$. The best fit for almost all models was obtained with $v_{\text {width }}=0.2 \mathrm{~km} \mathrm{~s}^{-1}, i=55^{\circ}$, and $R_{\text {out }}=180 \mathrm{AU}$, except for the free-falling disk model, where $R_{\text {out }}=130 \mathrm{AU}$. Since we do not have a way to constrain the temperature and column density gradients, we assume the observed emission is optically thin, and set the density as a constant and adjust the temperature gradient to match the observed spectral profile.

As discussed in Sect. 3.2, we suspect the presence of absorbing material along the line of sight of VLA1623, which would affect the observed emission and spectral profile, and thus influence the model fitting. From the data presented here we can not be completely certain of the characteristics of the absorbing material. Thus, we adjust the model velocities, peak opacity, and widths of the foreground clouds aiming to best fit the observed spectral profile. We assume that the foreground clouds only absorb with opacity following a Gaussian-like profile of the form

$\tau(v) \propto \exp \left(-0.5\left(\frac{v-v_{0}}{\sigma}\right)^{2}\right)$.

By adjusting the absorbing material parameters to fit the observed spectral profile, we find that the best results are obtained by introducing three absorbing layers, one corresponding to the envelope at $4.0 \mathrm{~km} \mathrm{~s}^{-1}$, and two corresponding to possible foreground clouds at 1.6 and $3.0 \mathrm{~km} \mathrm{~s}^{-1}$. The characteristics of the absorbing material for each model are listed in Table 3.

To study the kinematical structure of the disk, we modeled five cases: (1) free-falling disk; (2) free-falling outer disk plus inner Keplerian disk; (3) conservation of angular momentum (AM); (4) infalling $\left(v \propto R^{-1}\right)$ outer envelope plus inner Keplerian disk; and (5) pure Keplerian disk. For Cases 2, 3, and 4, we define a critical radius $R_{\text {crit }}$ parameter ( $1 \mathrm{AU} \leq R_{\text {crit }}<$ $R_{\text {out }}$ ) that defines the radius at which the transition from one velocity structure to the other occurs. Figure 5 illustrates the velocity structure distribution of each of the modeled cases. In Sect. 4.3 we briefly describe each model and the results obtained.

Figure 6 presents the best fitting results for the five cases modeled. We overlay the observed and modeled spatial distribution, spectral profile, and image-space PV diagram. Model spectral profiles are shown both with and without foreground absorption. The image-space PV diagram for each model was constructed along the disk's major axis. The best fit parameters obtained for each of the models are shown in Fig. 6 and listed in Table 2. To determine the best fit model of all five cases examined, we first compared each model and observations by eye and then through the residuals obtained by subtracting the channel maps of the observations and model. We deemed the model with the least residual the best fit (discarding the filaments to the north and south). Appendix B shows the channel map for each model, compared with observations and the corresponding residual.

\subsection{Model results}

Free falling disk: in the very early stages of star formation, the envelope of a protostar is thought to be under free fall collapse. Thus, if there was any disk-like structure around the protostar at this stage, its motion would be that of free fall as well, i.e. a freefalling disk (Fig. 5). In our model, we study a free-falling disk 

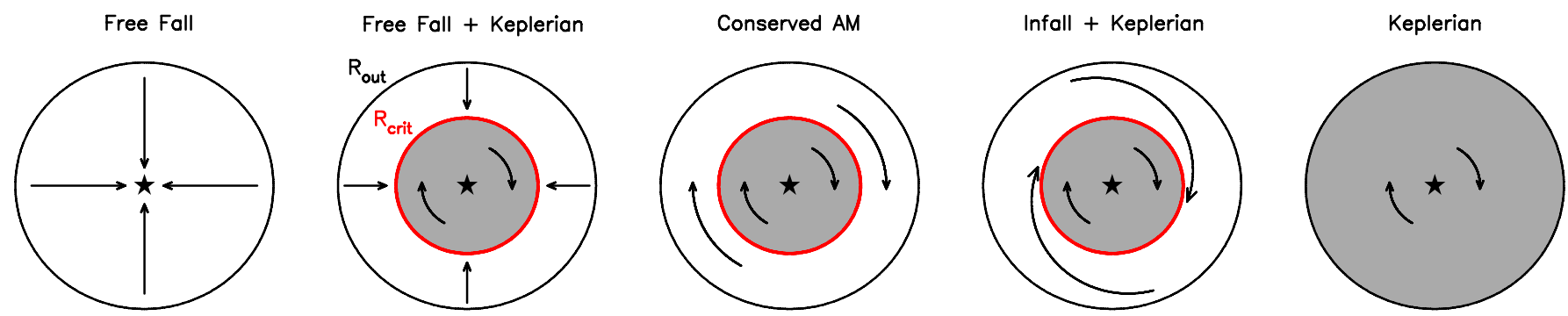

Fig. 5. Cartoon illustrating the velocity structure distribution in the disk, shown here face-on, for each model examined.

Table 2. Best fit thin disk model results.

\begin{tabular}{lccccc}
\hline \hline Parameter & Free fall & Free fall + Keplerian & Conserved AM & Infall + Keplerian & Keplerian \\
\hline$i$ (degrees) & 55 & 55 & 55 & 55 & 55 \\
$R_{\text {out }}(\mathrm{AU})$ & 130 & 180 & 180 & 180 & 180 \\
$R_{\text {crit }}(\mathrm{AU})$ & $\ldots$ & 150 & 20 & 150 & $\ldots$ \\
\hline
\end{tabular}

Table 3. Absorbing material for best fit thin disk model.

\begin{tabular}{|c|c|c|c|c|c|}
\hline & Free fall & Free fall + Keplerian & Conserved AM & Infall + Keplerian & Keplerian \\
\hline \multicolumn{6}{|l|}{ Envelope } \\
\hline velocity $\left(\mathrm{km} \mathrm{s}^{-1}\right)$ & 4.0 & 4.0 & 4.0 & 4.0 & 4.0 \\
\hline opacity & 7.0 & 7.0 & 7.0 & 7.5 & 7.0 \\
\hline width $\left(\mathrm{km} \mathrm{s}^{-1}\right)$ & 0.9 & 0.99 & 0.99 & 0.99 & 0.99 \\
\hline \multicolumn{6}{|l|}{ Foreground 1} \\
\hline velocity $\left(\mathrm{km} \mathrm{s}^{-1}\right)$ & 1.6 & 1.6 & 1.6 & 1.6 & 1.6 \\
\hline opacity & 5.0 & 5.0 & 5.0 & 5.0 & 5.0 \\
\hline width $\left(\mathrm{km} \mathrm{s}^{-1}\right)$ & 0.89 & 0.64 & 0.62 & 0.64 & 0.64 \\
\hline \multicolumn{6}{|l|}{ Foreground 2} \\
\hline velocity $\left(\mathrm{km} \mathrm{s}^{-1}\right)$ & 3.0 & 3.0 & 3.1 & 3.05 & 3.0 \\
\hline opacity & 0.39 & 0.29 & 0.29 & 0.29 & 0.29 \\
\hline width $\left(\mathrm{km} \mathrm{s}^{-1}\right)$ & 0.39 & 0.34 & 0.34 & 0.34 & 0.34 \\
\hline
\end{tabular}

structure where all motions are confined to the plane of the disk. The velocity structure within the disk structure is described as

$v_{\mathrm{ff}}^{r}=\sqrt{\frac{2 G M_{*}}{R}}$

In Fig. 6, the top row shows the thin disk model under free fall. Figure B.1 shows the channel map of the model and the residual. It is clear that the pure free fall case does not fit the observed $\mathrm{C}^{18} \mathrm{O}$ emission, since the velocity gradient is along the outflow direction, which is not the case in the observations. In addition, the wings in the spectral profile of this model are broad compared to the observed spectral profile.

Free fall plus Keplerian rotation: this case represents the formation of a rotationally supported disk in a young protostar whose outer disk is in free fall collapse (Fig. 5). To describe the velocity structure transition, we use the parameter $R_{\text {crit }}$, where $R<R_{\text {crit }}$ produces

$v_{\mathrm{rot}}^{\phi}=\sqrt{\frac{G M_{*}}{R}}$

and $R \geq R_{\text {crit }}$ is described by Eq. (2).

For this case, we experimented with different critical radii $R_{\text {crit }}$ to determine if the observed emission is a combination of Keplerian rotation and free fall (Fig. 7). We find that the free fall region ( $\left.R>R_{\text {crit }}\right)$ is clearly visible unless $R_{\text {crit }}$ approximates $R_{\text {out }}$. Similar to the pure free fall case, the outer free fall region produces a velocity gradient along the outflow direction, which is visible in the PV diagram (Fig. 7); however, we do not observe any velocity gradient along the outflow direction in the $\mathrm{C}^{18} \mathrm{O}$ structure (Fig. 2, right). In addition, the $\mathrm{C}^{17} \mathrm{O}$ (3-2) emission observed with SMA (Murillo \& Lai 2013) shows a velocity gradient indicative of rotation, thus the outer part of the observed structure is not under free fall. Figures 7 and B. 2 compare this model and the observations in detail, which show that only at $R_{\text {crit }}=150 \mathrm{AU}$ does the model closely approximate the observations.

Conserved Angular Momentum (AM): this case examines whether VLA1623A's $\mathrm{C}^{18} \mathrm{O}$ structure is described well by rotation with conserved angular momentum (Fig. 5), for example if the initial angular momentum of the cloud were conserved during collapse. The velocity structure in the inner region $R<R_{\text {crit }}$ is given by Eq. (3), while at $R \geq R_{\text {crit }}$ it is described by

$v_{\mathrm{AM}}^{\phi}=C \sqrt{\frac{G M_{*}}{R_{\text {crit }}}} \frac{R_{\text {crit }}}{R}$

where $\mathrm{C}$ is a constant indicating the increase in the Keplerian velocity at $R_{\text {crit }}$.

For this model, we find that $C=2$ with $R_{\text {crit }}=20$ AU provides the closest fit to the observations. In comparison to the other cases examined, this model produces broader wings and higher velocities at smaller radii, effects visible in the spectral profile and the PV diagram, respectively (Fig. 6). Additionally, from the spectral profile shown in Fig. 6, it is seen that this model does not correctly reproduce the blueshifted peak. Changing the $C$ parameter does not resolve this issue, and changing the 

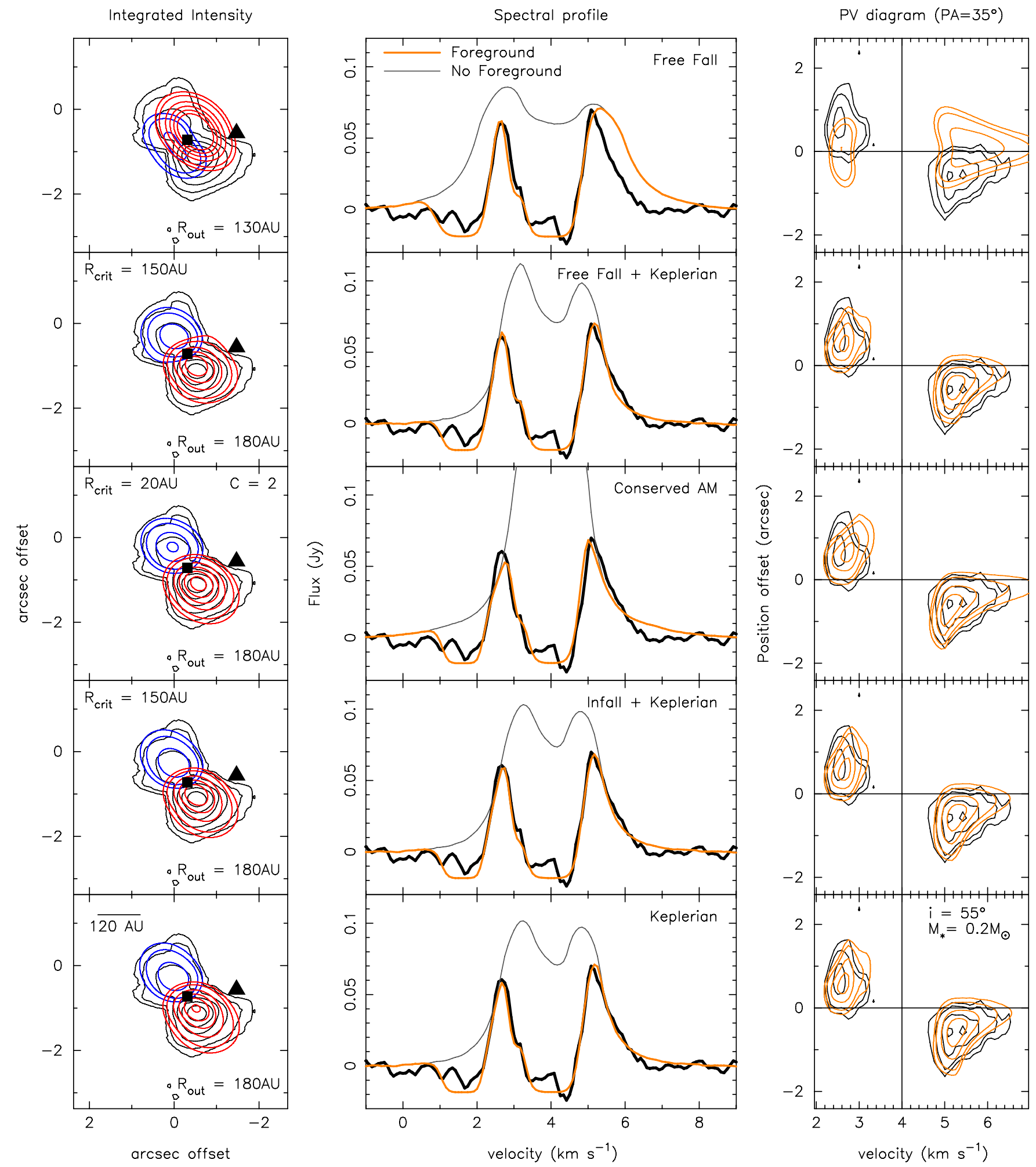

Fig. 6. Comparison of $\mathrm{C}^{18} \mathrm{O}$ observations (black lines) with a thin disk model (colored lines) that includes foreground components. The left column shows moment 0 maps with the red and blue lines showing red and blueshifted emission, respectively. Source positions are marked as in Fig. 2. The middle column compares the spectral profiles with and without foreground. The right column presents the image-space PV diagrams. The models shown here have two foreground components (at 1.6 and $3.0 \mathrm{~km} \mathrm{~s}^{-1}$ ) and the resolved-out envelope component (at $4.0 \mathrm{~km} \mathrm{~s}{ }^{-1}$ ). Contours both for observations and models are the same as those in Fig. 2. $M_{*}$ and $i$ for all models are shown in the right panel.

line width also broadens the wings. Furthermore, even though from the intensity integrated map the spatial distribution appears to fit the observations, inspecting the channel map residuals shows that the model does not quite agree with the observations
(Fig. B.3). Thus we determine that this scenario does not match the observations.

Infall plus Keplerian rotation: this case is similar to the free fall plus Keplerian case, but with the outer part of the 

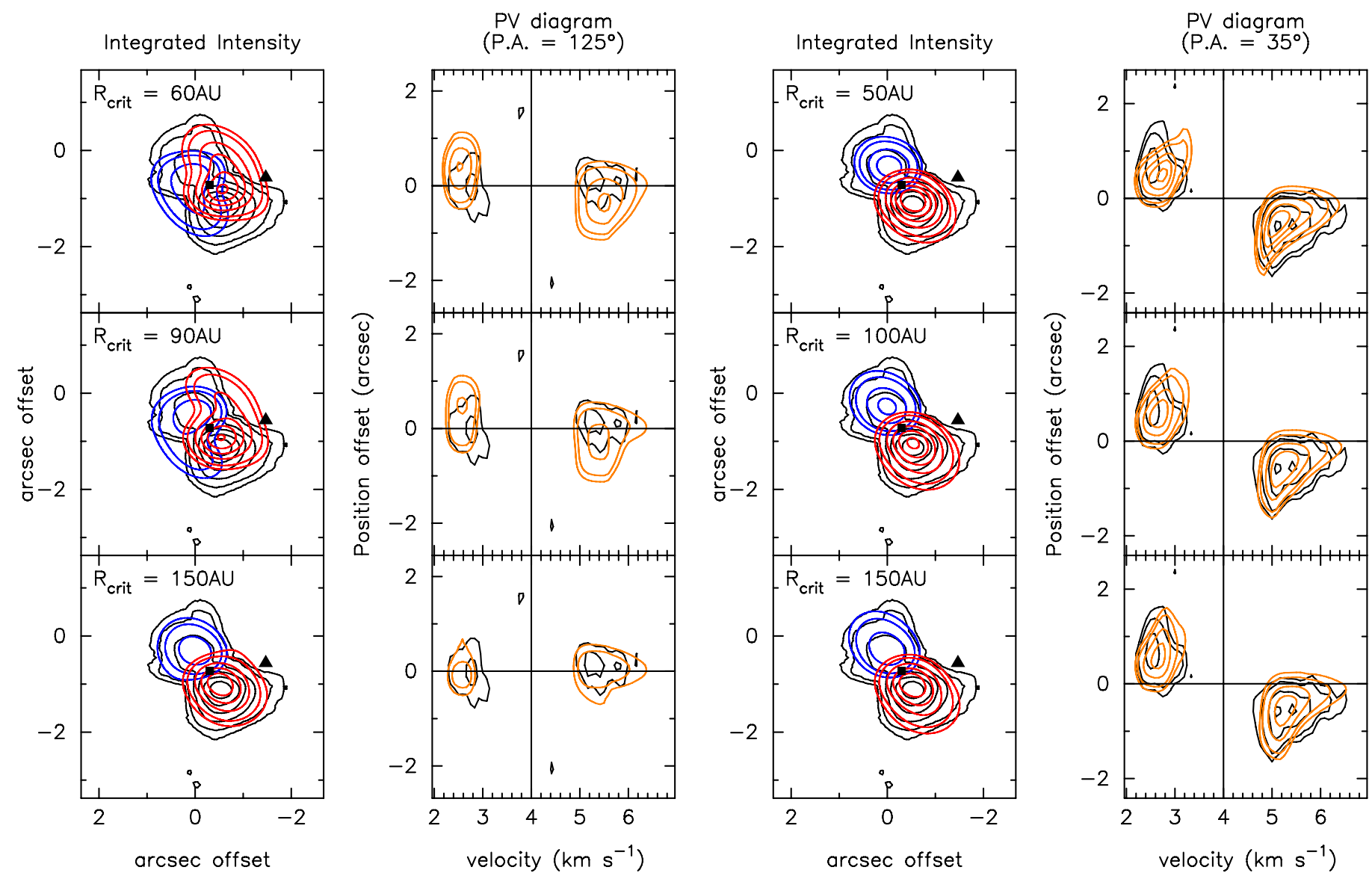

Fig. 7. Free fall plus Keplerian case for three different $R_{\text {crit. }}$ The best fit model (color) to the data (black) is obtained as $R_{\text {crit }}$ approaches $R_{\text {out }}$. Right panels show PV diagram along outflow direction $\left(\mathrm{PA}=125^{\circ}\right)$. All contours and the model at $R_{\text {crit }}=150 \mathrm{AU}$ are the same as in Fig. 6 .

envelope infalling onto the rotationally supported disk (Fig. 5). We consider this model a possibility from the results of the UVspace PV diagram (Fig. 4), which shows an inner region under Keplerian rotation and an outer region of infall, with a critical radius of $\sim 50 \mathrm{AU}$. Thus, for the inner rotating region (i.e. $R<R_{\text {crit }}$ ) the velocity structure is described by Eq. (3), and for the outer infalling region $\left(R \geq R_{\text {crit }}\right)$ it is given by

$v_{\text {infall }}^{r}=\sqrt{\frac{G M_{*}}{R_{\text {crit }}}} \frac{R_{\text {crit }}}{R}$.

As in the free fall plus Keplerian case, we ran the model with different critical radii $R_{\text {crit }}$ to determine how far each of the velocity structures extends (Fig. 8). Similar to the free fall plus Keplerian case, we find that the best fit occurs as $R_{\text {crit }}$ approaches $R_{\text {out }}$, with the best fit having $R_{\text {crit }}=150$ AU (Figs. 6 and 8). The discrepancy between the $R_{\text {crit }}$ obtained from the modeling (150 AU) and that of the UV-space PV diagram from observations (50 AU) may be due to the low-velocity points being greatly affected by optical depth and absorption of the envelope. Keplerian rotation out to at least $150 \mathrm{AU}$ represents a best fit scenario for the observed $\mathrm{C}^{18} \mathrm{O}$ emission. Because of the large $R_{\text {crit }}$, it is difficult to distinguish this scenario from the following case. In Fig. B.4 the channel map comparison and residual between this model and the observations are shown.

Pure Keplerian rotation: in this case we model a Keplerian disk around a central protostar (Fig. 5). This velocity structure

Fig. 8. Infall plus Keplerian case for three different $R_{\text {crit. }}$. The best fit model (color) to the data (black) is obtained as $R_{\text {crit }}$ approaches $R_{\text {out }}$. Right panels show PV diagrams along the disk's major axis $\left(\mathrm{PA}=35^{\circ}\right)$. All contours and the model at $R_{\text {crit }}=150 \mathrm{AU}$ are the same as in Fig. 6.

has been observed starting in Class I protostars and is common among Class II protostars. The velocity structure is given by Eq. (3).

We find this case to fit the observed $\mathrm{C}^{18} \mathrm{O}$ emission well, as the model agrees with the observations spatially, in the spectral profile and in the image-space PV diagram (Fig. 6). However, it is difficult to determine whether this scenario is considerably better than the infall plus Keplerian case. We discuss this further in the next section. In Fig. B.5 the channel map comparison and residual between this model and the observations are shown.

\section{Discussion}

Our ALMA observations of VLA1623 reveal that the Class 0 component of this system, VLA1623A, has a disk structure in $\mathrm{C}^{18} \mathrm{O}(2-1)$ with an outer radius of $180 \mathrm{AU}$. Thin disk modeling with the addition of foreground indicates that the disk structure is most certainly Keplerian out to a radius of $150 \mathrm{AU}$. For the outer $30 \mathrm{AU}$, it is uncertain whether the velocity structure may be undergoing infall $\left(v \propto R^{-1}\right)$ or pure Keplerian rotation. This uncertainty is due to the low signal-to-noise ratio $(\mathrm{S} / \mathrm{N})(5 \sigma)$ in the outer edges of the observed disk structure, while the inner regions of the disk have a higher $\mathrm{S} / \mathrm{N}$ (10 to $25 \sigma)$, thus a better and more reliable fit is obtained in the inner parts of the observed structure. The presence of such a large Keplerian disk raises the 
Table 4. Class 0 sources with disk.

\begin{tabular}{lcccc}
\hline \hline Parameter & NGC 1333 IRAS4A2 $^{a}$ & L1527 & VLA1623A & Ref. \\
\hline$i$ (degrees) & 10.7 & $\sim 85$ & 55 & $1,2,3$ \\
$R_{\text {out }}(\mathrm{AU})$ & 310 & 90 & 180 & $1,2,3$ \\
$M_{*}\left(M_{\odot}\right)$ & $0.08 \pm 0.02$ & $0.19 \pm 0.04$ & $0.22 \pm 0.02$ & $1,2,3$ \\
$M_{\text {env }}{ }^{b}\left(M_{\odot}\right)$ & 5.6 & 0.9 & 0.8 & 4,5 \\
$M_{*} / M_{\text {env }}$ & 0.01 & 0.2 & 0.28 & $\ldots$ \\
$T_{\text {bol }}(\mathrm{K})$ & 51 & 44 & 10 & $1,5,3$ \\
$L_{\text {bol }}\left(L_{\odot}\right)$ & $1.9 \pm 0.9$ & 1.97 & $1.1 \pm 0.2$ & $1,2,6$ \\
$L_{\text {submm }} / L_{\text {bol }}{ }^{c}(\%)$ & 3.6 & 0.8 & 1.2 & $\ldots$ \\
\hline
\end{tabular}

Notes. ${ }^{(a)} T_{\text {bol }}$ and $L_{\text {bol }}$ parameters are for NGC 1333 IRAS4A, Choi et al. (2010) assume IRAS4A2 contributes half of the $L_{\mathrm{bol}} \cdot{ }^{(b)} M_{\mathrm{env}}$ is the total envelope mass of each system. ${ }^{(c)} L_{\text {submm }} / L_{\text {bol }}>0.5 \%$ is characteristic of Class 0 source (Froebrich 2005; André et al. 1993).

References. (1) Choi et al. (2010); (2) Tobin et al. (2012); (3) this work; (4) Froebrich (2005); (5) Kristensen et al. (2012); (6) Murillo \& Lai (2013).

question of how disks are formed in the early stages of protostellar evolution.

Regardless of the model, neither the infall plus Keplerian case nor the pure Keplerian case reproduces the weak filamentlike structures to the north of the blueshifted emission $(v=2.3$ to $\left.2.47 \mathrm{~km} \mathrm{~s}^{-1}\right)$ and south of the redshifted emission $(v=4.8$ to $4.97 \mathrm{~km} \mathrm{~s}^{-1}$ ) (Figs. 2, B.4, and B.5). These structures may be produced by a disk wind (Klaassen et al. 2013) or material entrained by the outflow.

The idealized non-magnetized isothermal sphere collapse model of Terebey et al. (1984) suggests that disks may form during the early protostellar stages. As previously mentioned, under these conditions, the disk's centrifugal radius $R_{\mathrm{c}}$ is expected to grow proportional to $t^{3}$, where $t$ denotes the time since collapse. To calculate the time since collapse for $R_{\mathrm{c}}=150 \mathrm{AU}$, we use the equation from Belloche (2013)

$R_{\mathrm{c}}(\mathrm{AU})=39\left(\frac{\Omega}{4 \times 10^{-14} \mathrm{rad} \mathrm{s}^{-1}}\right)^{2}\left(\frac{a}{0.2 \mathrm{~km} \mathrm{~s}^{-1}}\right)\left(\frac{m_{*+d}}{1 M_{\odot}}\right)^{3}$

where $\Omega$ is the initial cloud core rotation rate, $a$ is the sound speed, and $m_{*+d}=0.975 \frac{a^{3}}{G} t$. Assuming VLA1623 has a rotation rate $\Omega=4 \times 10^{-14} \mathrm{rad} \mathrm{s}^{-1}$, and the sound speed in the core is of $a=0.2 \mathrm{~km} \mathrm{~s}^{-1}$, we obtain $t=8.5 \times 10^{5} \mathrm{yr}$ for a centrifugal radius of $150 \mathrm{AU}$. However, the old age obtained through this method is inconsistent with the expected age from $T_{\text {bol }}$ (Ladd et al. 1998) and the estimated Class 0 lifetime obtained by Evans et al. (2009), which is on the order of $10^{5} \mathrm{yr}$, although they conceded that for $\rho$ Ophiuchus the lifetime is $4 \times 10^{4} \mathrm{yr}$, an order of magnitude lower than the age calculated above. Furthermore, based on outflow observations, VLA1623's dynamical timescale is between 0.2 and $2.5 \times 10^{4} \mathrm{yr}$ (André et al. 1990; Nakamura et al. 2011). This indicates that there are other factors that enhance the formation of the disk. Possible factors that can influence disk formation may be fragmentation, turbulence, or the misalignment of the magnetic field and rotation axis. For the first factor, fragmentation, there is little work done on how fragmentation can enhance or hinder disk formation, with most work focusing on how a disk fragments. However, there may be a possible relation given that two of the three Class 0 sources reported as having rotationally supported disks are confirmed multiples (see Sect. 1 and below). The introduction of turbulence and its effect on disk formation from low to high masses has been studied by Seifried et al. (2013), finding that turbulence can encourage disk formation even when strong magnetic fields are present. Nakamura et al. (2011) studied the outflow generated turbulence in $\rho$ Ophiuchus' main cloud, concluding that outflows can influence the dense cores significantly. However, from Nakamura et al. (2011) or this work, there is not enough information to determine the degree of influence that turbulence has on the formation of VLA1623A's disk. At present we can neither examine further nor rule out the role of turbulence. The last factor, magnetic field misalignment, is considered in-depth below.

Magnetic fields are expected to influence the formation of protostellar disks in the early stages of protostar formation. It it thus of interest to look into the magnetic field configuration of VLA1623. Holland et al. (1996) observed $800 \mu$ m polarization with the JCMT, finding a $2 \%$ polarization and a magnetic field perpendicular to the outflow direction. Hull et al. (2013b) also observed the field to be perpendicular to the outflow down to 2 " resolution with CARMA observations. Murillo \& Lai (2013) carried out polarization observations with the SMA (compact configuration, resolution $\sim 1.5^{\prime \prime}$ ) but found no significant detection. From these results, we infer that the magnetic field towards VLA1623 is not aligned with the rotation axis of VLA1623A's disk, and the field strength may be low. Krumholz et al. (2013) find that rotationally supported disks should form as early as the Class 0 stages with sizes of $100 \mathrm{AU}$ or larger when the magnetic field direction and the disk's rotational axis misalignment are large and the magnetic field strength is low. The presence of a fairly large rotationally supported disk around VLA1623A is consistent with the Krumholz et al. (2013) predictions, given VLA1623A's misalignment of $84^{\circ}$ between magnetic field direction and rotational axis (Hull et al. 2013a). In addition, the disk size is sensitive to the initial cloud density profile, with centrally concentrated profiles favoring larger disks. Moreover, the discrepancy between magnetized models of disk formation and VLA1623A's disk may be due to the sink particle parameters used to represent the forming protostar in the models (Machida et al. 2013).

Two other Class 0 sources have been reported as presenting a Keplerian disk structure: NGC 1333 IRAS4A2 and L1527. The characteristics of these sources are listed in Table 4 along with VLA1623A's characteristics. It should be noted that the parameters for NGC 1333 IRAS4A2 are obtained under the assumption that this source contributes half of the bolometric luminosity and that both sources in the NGC 1333 IRAS4A binary have the same bolometric temperature. Thus, in comparisson, VLA1623A is noticeably younger than L1527, based on 
the bolometric temperature and luminosity ratio. On the other hand, we cannot determine the relative evolutionary stages of VLA1623A and NGC 1333 IRAS4A2 with certainty. We can assume, however, that VLA1623A is younger than NGC 1333 IRAS4A2 based on the bolometric temperature. It must be noted, though, that the inclination angle of a protostar can affect the calculated parameters and thus affect the derived evolutionary parameters. Given that all three sources have different inclination angles, this may well affect the comparison. However, it is very possible that VLA1623A's disk is the youngest disk among the three sources, given that VLA1623A is still deeply embedded and shows no emission shortward of $24 \mu \mathrm{m}$.

\section{Conclusions}

We have presented ALMA Cycle 0 Early Science Band 6 extended configuration observations of $\mathrm{C}^{18} \mathrm{O}(2-1)$ and continuum towards VLA1623. From these observations, we found three continuum sources that are consistent with previous observations and $\mathrm{C}^{18} \mathrm{O}$ emission centered at VLA1623A with signatures of rotation and an outer radius of 180 AU. Through PV diagram analysis and modeling of the observed $\mathrm{C}^{18} \mathrm{O}$, we determine that the emission traces a Keplerian disk out to $150 \mathrm{AU}$ around a $0.2 \pm 0.02 M_{\odot}$ protostar. The weak magnetic field and its misalignment with the disk's rotational axis may have increased the chances of disk formation at such an early stage. There may also be the possibility that fragmentation played a role in the early disk formation, but this is unclear from the results obtained here. Comparison of evolutionary indicators of VLA1623A with those of the other Class 0 sources showing indications of Keplerian disks suggest that VLA1623A's disk may be the youngest among the Class 0 Keplerian disks. Our results show that disks, and more precisely rotationally supported or Keplerian disks, can be formed in the Class 0 stage of protostellar evolution even with fairly large radii. However, our results also hint that the environmental factors play a larger role than evolutionary stage in the formation of disks.

Acknowledgements. This paper made use of the following ALMA data: ADS/JAO.ALMA\# 2011.0.00902.S. ALMA is a partnership of ESO (representing its member states), NSF (USA), and NINS (Japan), together with NRC (Canada) and NSC and ASIAA (Taiwan), in cooperation with the Republic of Chile. The Joint ALMA Observatory is operated by ESO, AUI/NRAO, and NAOJ. The data was obtained by N.M.M. while she was a Master student at National Tsing Hua University, Taiwan, under the supervision of S.P.L. S.P.L. acknowledges support from the National Science Council of Taiwan with Grants NSC 98-2112-M-007-007-MY3 and NSC 101-2119-M-007-004. Astrochemistry in Leiden is supported by the Netherlands Research School for Astronomy (NOVA) by a Spinoza grant and grant 614.001.008 from the Netherlands Organization for Scientific Research (NWO), and by the European Community's Seventh Framework Program FP7/2007-2013 under grant agreement 238258 (LASSIE).

\section{References}

André, P., Martin-Pintado, J., Despois, D., \& Montmerle, T. 1990, A\&A, 236, 180

André, P., Ward-Thompson, D., \& Barsony, M. 1993, ApJ, 406, 122

Belloche, A. 2013, EAS Pub. Ser., 62, 25

Boogert, A. C. A., Hogerheijde, M. R., Ceccarelli, C., et al. 2002, ApJ, 570, 708 Brinch, C., Crapsi, A., Jørgensen, J. K., Hogerheijde, M. R., \& Hill, T. 2007, A\&A, 475, 915

Chen, X., Arce, H. G., Zhang, Q., et al. 2013, ApJ, 768, 110

Choi, M., Tatematsu, K., \& Kang, M. 2010, ApJ, 723, L34

Davidson, J. A., Novak, G., Matthews, T. G., et al. 2011, ApJ, 732, 97

Dzib, S. A., Loinard, L., Mioduszewski, A. J., et al. 2013, ApJ, 775, 63

Enoch, M. L., Corder, S., Dunham, M. M., \& Duchêne, G. 2009, ApJ, 707, 103

Enoch, M. L., Corder, S., Duchêne, G., et al. 2011, ApJS, 195, 21

Evans, II, N. J., Dunham, M. M., Jørgensen, J. K., et al. 2009, ApJS, 181, 321

Froebrich, D. 2005, ApJS, 156, 169

Girart, J. M., Rao, R., \& Marrone, D. P. 2006, Science, 313, 812

Guilloteau, S., \& Dutrey, A. 1998, A\&A, 339, 467

Guilloteau, S., Dutrey, A., \& Simon, M. 1999, A\&A, 348, 570

Hennebelle, P., \& Ciardi, A. 2009, A\&A, 506, L29

Hogerheijde, M. R. 2001, ApJ, 553, 618

Holland, W. S., Greaves, J. S., Ward-Thompson, D., \& André, P. 1996, A\&A, 309, 267

Hughes, A. M., Andrews, S. M., Espaillat, C., et al. 2009, ApJ, 698, 131

Hull, C. L. H., Plambeck, R. L., Bolatto, A. D., et al. 2013a, ApJ, 768, 159

Hull, C. L. H., Plambeck, R. L., Kwon, W., et al. 2013b [arXiv: 1310.6653]

Jørgensen, J. K., Schöier, F. L., \& van Dishoeck, E. F. 2002, A\&A, 389, 908

Jørgensen, J. K., Schöier, F. L., \& van Dishoeck, E. F. 2004, A\&A, 416, 603

Jørgensen, J. K., van Dishoeck, E. F., Visser, R., et al. 2009, A\&A, 507, 861

Klaassen, P. D., Juhasz, A., Mathews, G. S., et al. 2013, A\&A, 555, A73

Kristensen, L. E., van Dishoeck, E. F., Bergin, E. A., et al. 2012, A\&A, 542, A8

Krumholz, M. R., Crutcher, R. M., \& Hull, C. L. H. 2013, ApJ, 767, L11

Ladd, E. F., Fuller, G. A., \& Deane, J. R. 1998, ApJ, 495, 871

Li, Z.-Y., Krasnopolsky, R., \& Shang, H. 2011, ApJ, 738, 180

Loinard, L., Rodríguez, L. F., D’Alessio, P., Wilner, D. J., \& Ho, P. T. P. 2002, ApJ, 581, L109

Loinard, L., Torres, R. M., Mioduszewski, A. J., \& Rodríguez, L. F. 2008, ApJ, 675, L29

Lommen, D., Jørgensen, J. K., van Dishoeck, E. F., \& Crapsi, A. 2008, A\&A, 481,141

Loren, R. B. 1989, ApJ, 338, 902

Machida, M. N., Inutsuka, S.-I., \& Matsumoto, T. 2013, MNRAS, submitted [arXiv: 1307. 1747]

Mannings, V., \& Sargent, A. I. 1997, ApJ, 490, 792

Mardones, D., Myers, P. C., Tafalla, M., et al. 1997, ApJ, 489, 719

Mellon, R. R., \& Li, Z.-Y. 2008, ApJ, 681, 1356

Murillo, N. M., \& Lai, S.-P. 2013, ApJ, 764, L15

Nakamura, F., Kamada, Y., Kamazaki, T., et al. 2011, ApJ, 726, 46

Qi, C., Ho, P. T. P., Wilner, D. J., et al. 2004, ApJ, 616, L11

Reipurth, B. 2000, AJ, 120, 3177

Rodriguez, D. R., Kastner, J. H., Wilner, D., \& Qi, C. 2010, ApJ, 720, 1684

Seifried, D., Banerjee, R., Pudritz, R. E., \& Klessen, R. S. 2013, MNRAS, 432, 3320

Takakuwa, S., Saito, M., Lim, J., et al. 2012, ApJ, 754, 52

Terebey, S., Shu, F. H., \& Cassen, P. 1984, ApJ, 286, 529

Tobin, J. J., Hartmann, L., Chiang, H.-F., et al. 2012, Nature, 492, 83

van Kempen, T. A., van Dishoeck, E. F., Salter, D. M., et al. 2009, A\&A, 498, 167

Yen, H.-W., Takakuwa, S., Ohashi, N., \& Ho, P. T. P. 2013, ApJ, 772, 22

Yu, T., \& Chernin, L. M. 1997, ApJ, 479, L63 


\section{Appendix A: VLA1623W}

In our ALMA observations, we detect VLA1623W in continuum (see Sect. 3.1 and Fig. 1) and in $\mathrm{C}^{18} \mathrm{O}$ line emission (Fig. A.1), which was previously undetected with SMA observations. The emission is weak, peaking at $5 \sigma$ in the channel maps and at $13 \sigma$ in the intensity integrated map, and it has a velocity range of about -0.5 to $1 \mathrm{~km} \mathrm{~s}^{-1}$. Noteworthy is VLA1623W's apparent systemic velocity $v_{\text {Isr }}$ between 0 and $1 \mathrm{~km} \mathrm{~s}^{-1}$, which differs from VLA1623A's systemic velocity by 3 to $4 \mathrm{~km} \mathrm{~s}^{-1}$. VLA1623W's $v_{\mathrm{lsr}}$ is difficult to determine with certainty since the emission appears to be strongly affected by foreground material (see Sect. 3.2) and the low $\mathrm{S} / \mathrm{N}$ of the detection. Finally, no $\mathrm{C}^{18} \mathrm{O}$ emission was detected towards VLA1623B (Fig. 1, top left inset), which is consistent with previous $\mathrm{CO}$ depletion findings (Murillo \& Lai 2013).

The velocity gradient of VLA1623W's $\mathrm{C}^{18} \mathrm{O}$ emission appears to be consistent with a rotationally supported disk structure, and given that VLA1623W is classified as a Class I source (Murillo \& Lai 2013), this is very likely. However, since the emission is very close to the edge of the field of view (Fig. 1) and is affected by foreground absorption, we are limited in carrying out kinematical analysis of the emission.

Finally the large difference in systemic velocity from VLA1623A and B may suggest one of two scenarios. First, VLA1623W is not part of the system, but is instead a foreground or nearby source, that due to projection effects seems to be part of the system. However, Dzib et al. (2013) rule out the possibility of VLA1623W (or VLA1623B) being a foreground or background object due to its proper motion. Second, due to three-body interaction, VLA1623W was ejected from the closer binary of VLA1623A and B (Reipurth 2000). This possibility is very likely, given that VLA1623B may be a very recent formation, which would have caused the binary, now triple, to become unstable and eject one of the components. This ejection would cause VLA1623W to lose some of its envelope mass and appear more evolved, since one of the classification criteria is the envelope to central star mass ratio. The loss of mass would also make it more visible in the infrared and affect its evolution.

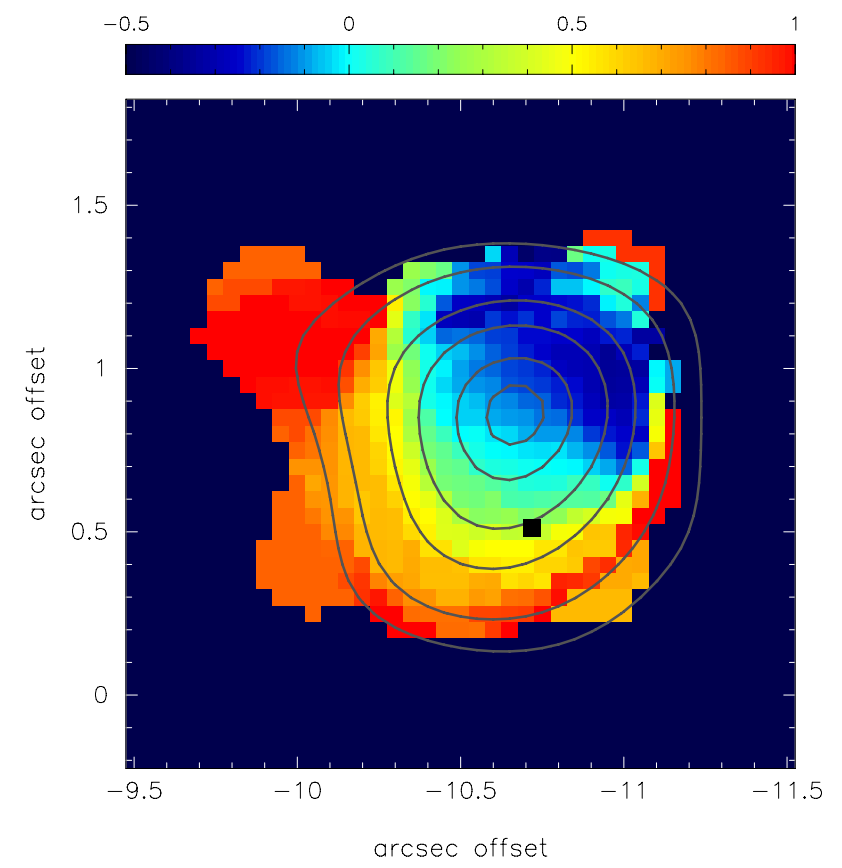

Fig. A.1. Integrated intensity (moment 0, contours) and velocity (moment 1 , halftone) maps of $\mathrm{C}^{18} \mathrm{O}(2-1)$ detection towards VLA1623W. The position of VLA1623W is marked with a black square. Contours are in steps of $3 \sigma, 5 \sigma, 8 \sigma, 10 \sigma, 12 \sigma$, and $13 \sigma$ with $\sigma=13 \mathrm{mJy}_{\text {beam }}^{-1}$.

\section{Appendix B: Comparison of thin disk models and observations}

In this appendix, we present the channel map comparison for each model and observations, as well as the residual obtained from subtracting the model from the observations. The figures presented below are a supplement to Fig. 6 in order to gauge the best-fitting model of the observed emission. All figures below show the blueshifted emission in the velocity range of 2.3 to $3 \mathrm{~km} \mathrm{~s}^{-1}$, while the redshifted emission is in the velocity range of 4.8 to $6.5 \mathrm{~km} \mathrm{~s}^{-1}$. 


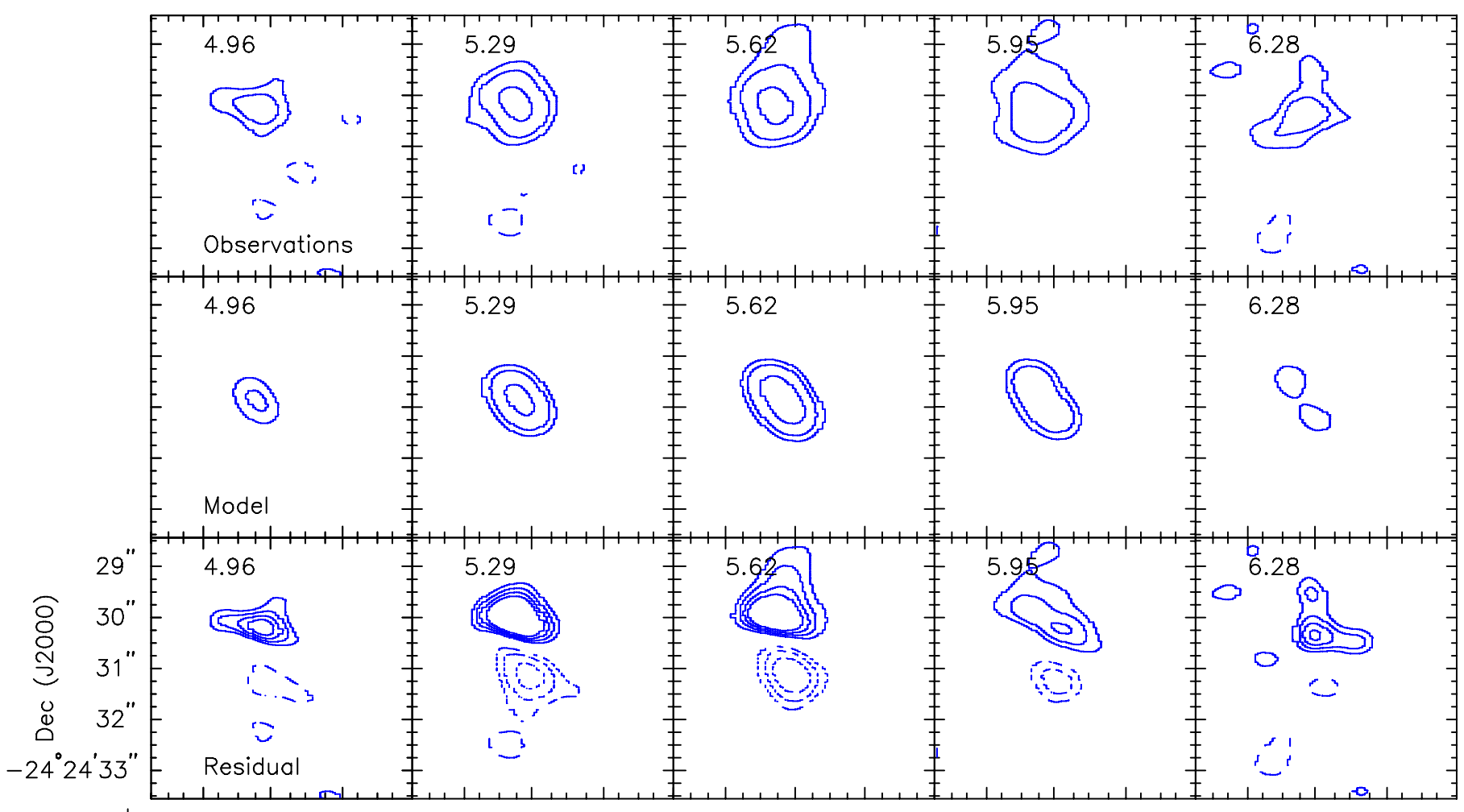

$16^{\mathrm{h}} 26^{\mathrm{m}} 26^{\mathrm{s}} .526^{\mathrm{s}} .426^{\mathrm{s}} .326^{\mathrm{s}} .2$

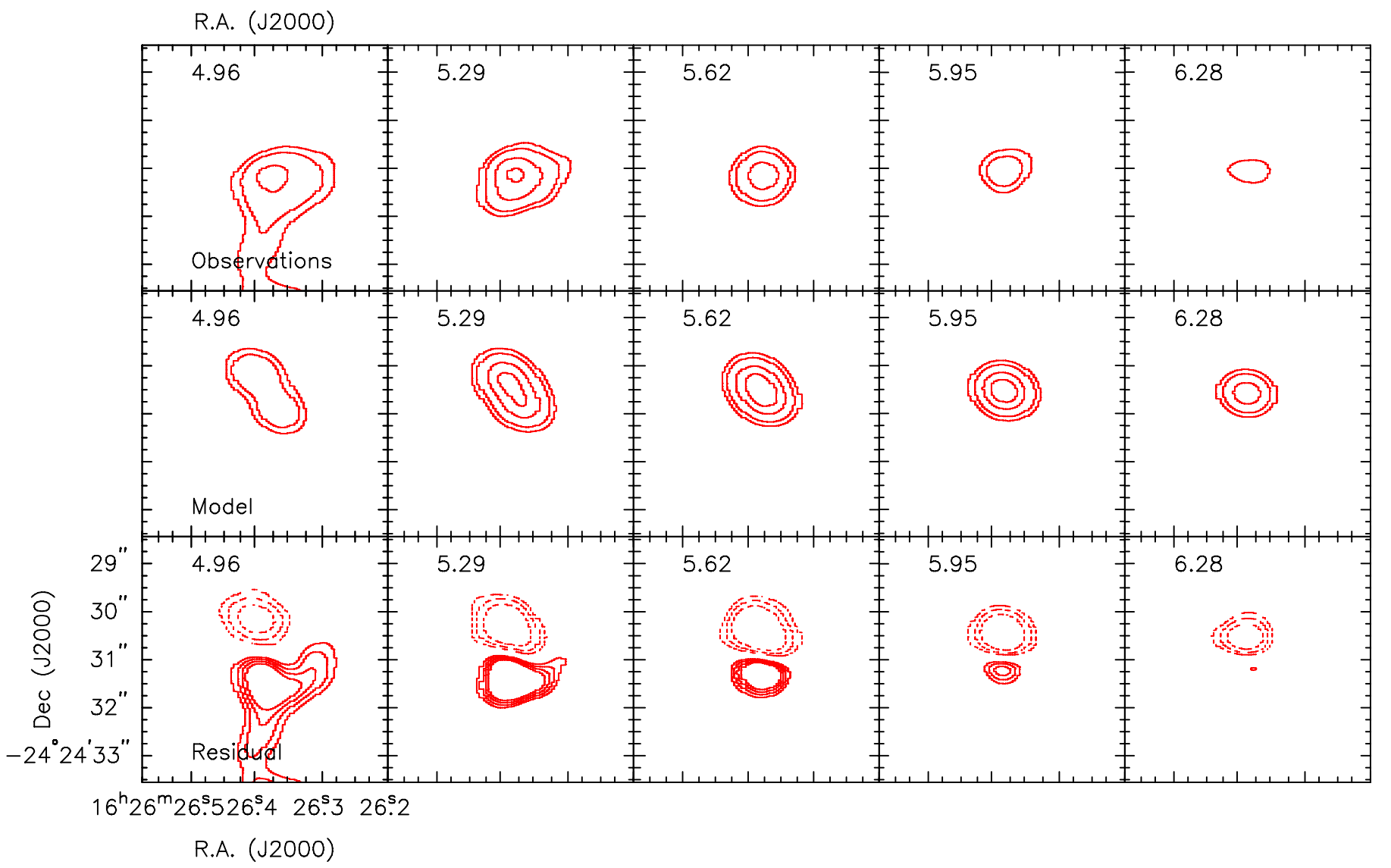

Fig. B.1. Channel maps of $\mathrm{C}^{18} \mathrm{O}$ observations (top), free falling disk model shown in Fig. 6 (middle) and residual (bottom panel). The blue contours show the blueshifted emission and the red contours the redshifted emission. Number labels on the top of each panel indicate the velocity of that channel. Channels are binned to a velocity resolution of $0.16 \mathrm{~km} \mathrm{~s}^{-1}$ for the blueshifted emission, and $0.33 \mathrm{~km} \mathrm{~s}^{-1}$ for the redshifted emission for better display. Contours are in steps of $3 \sigma, 5 \sigma, 10 \sigma, 15 \sigma, 20 \sigma$ and $25 \sigma$ for the observations and model channel maps, and $-8 \sigma,-5 \sigma,-3 \sigma, 3 \sigma$, $4 \sigma, 5 \sigma$ and $6 \sigma$ for the residual channel map, where $\sigma=19$ mJy beam $^{-1}$. 
N. M. Murillo et al.: A Keplerian disk around a Class 0 source: ALMA observations of VLA1623A

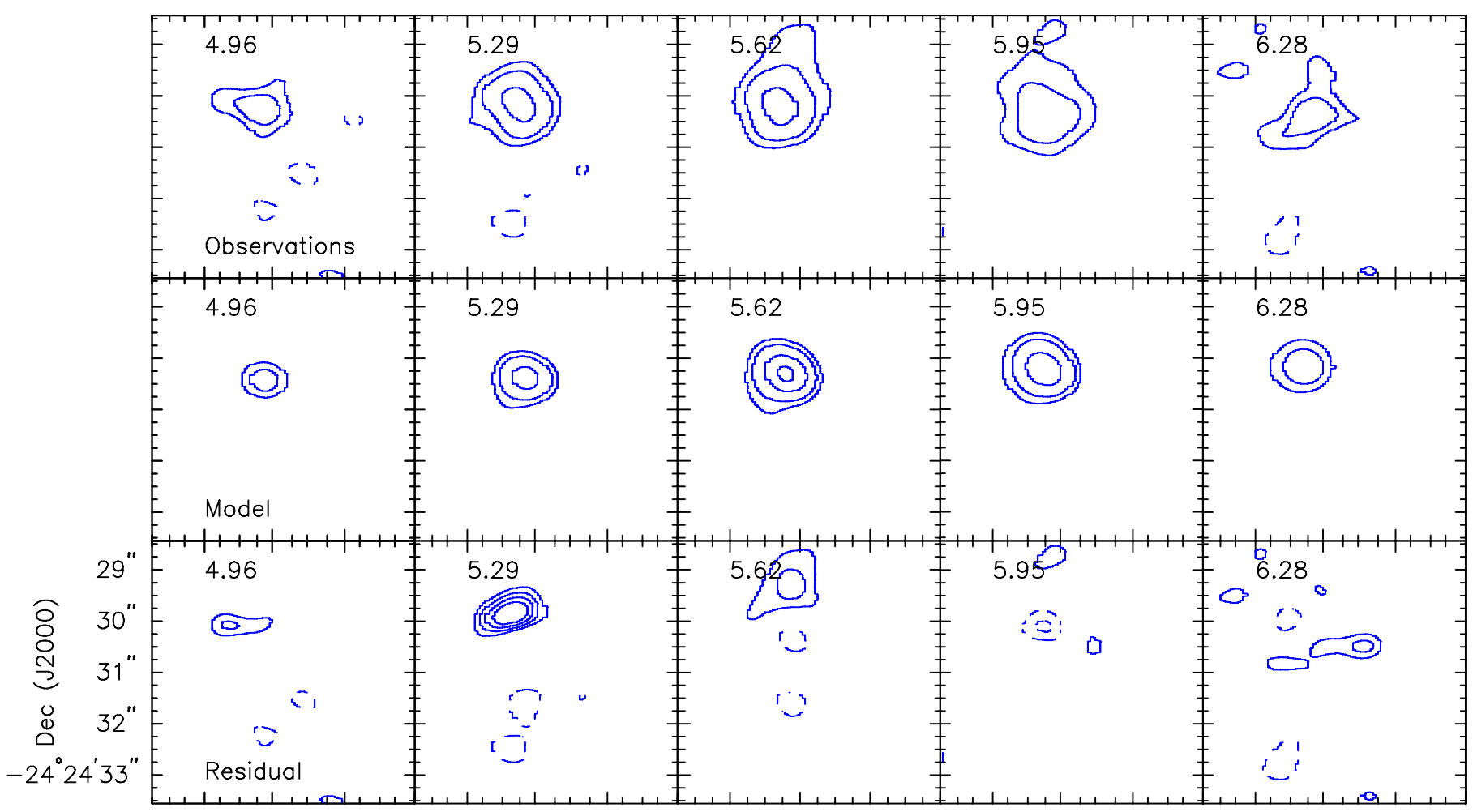

$16^{\mathrm{h}} 26^{\mathrm{m}} 26^{\mathrm{s}} .526^{\mathrm{s}} \cdot 426^{\mathrm{s}} \cdot 326^{\mathrm{s}} \cdot 2$

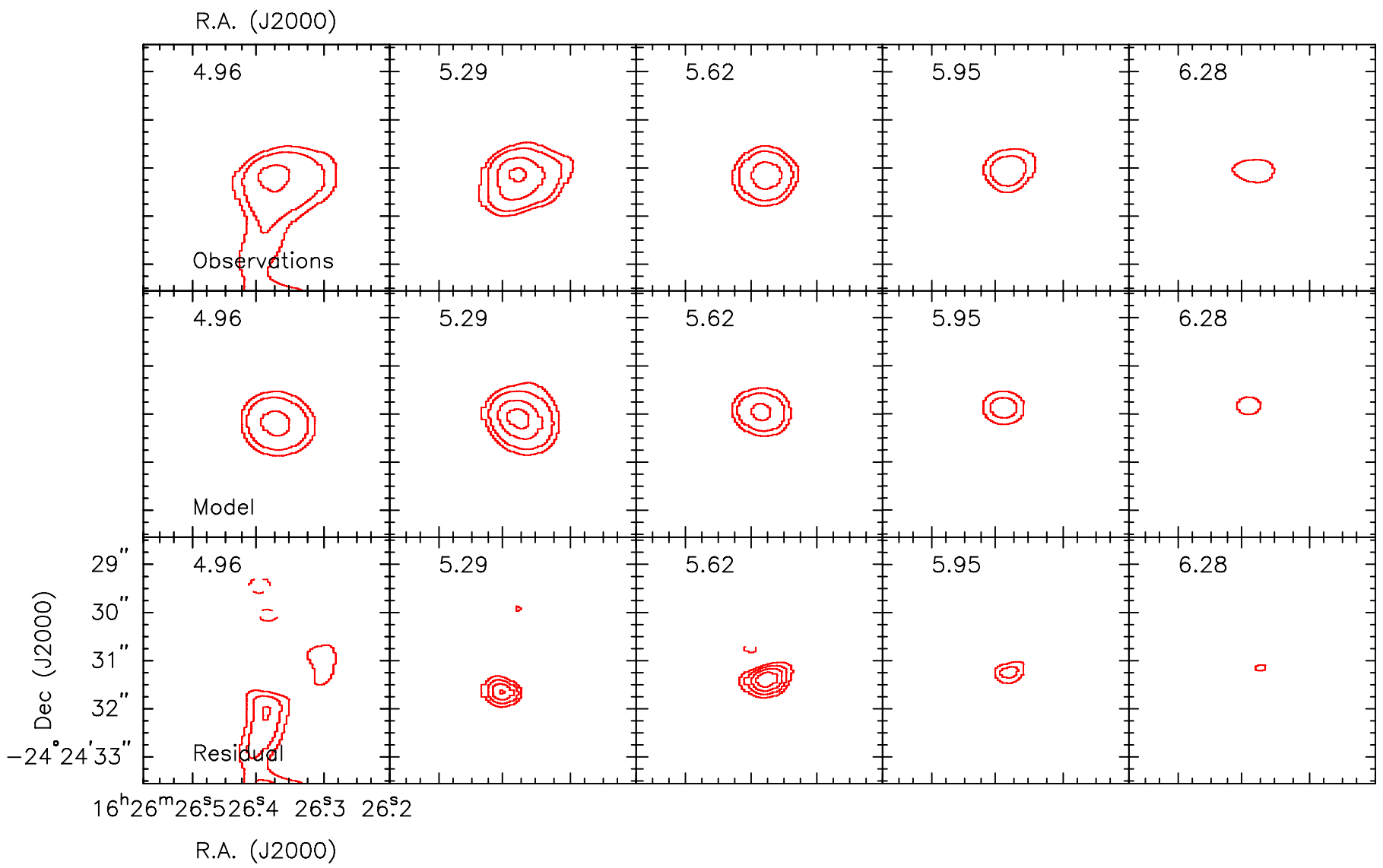

Fig. B.2. Same as Fig. B.1 but for the free fall plus Keplerian disk model shown in Fig. 6. 


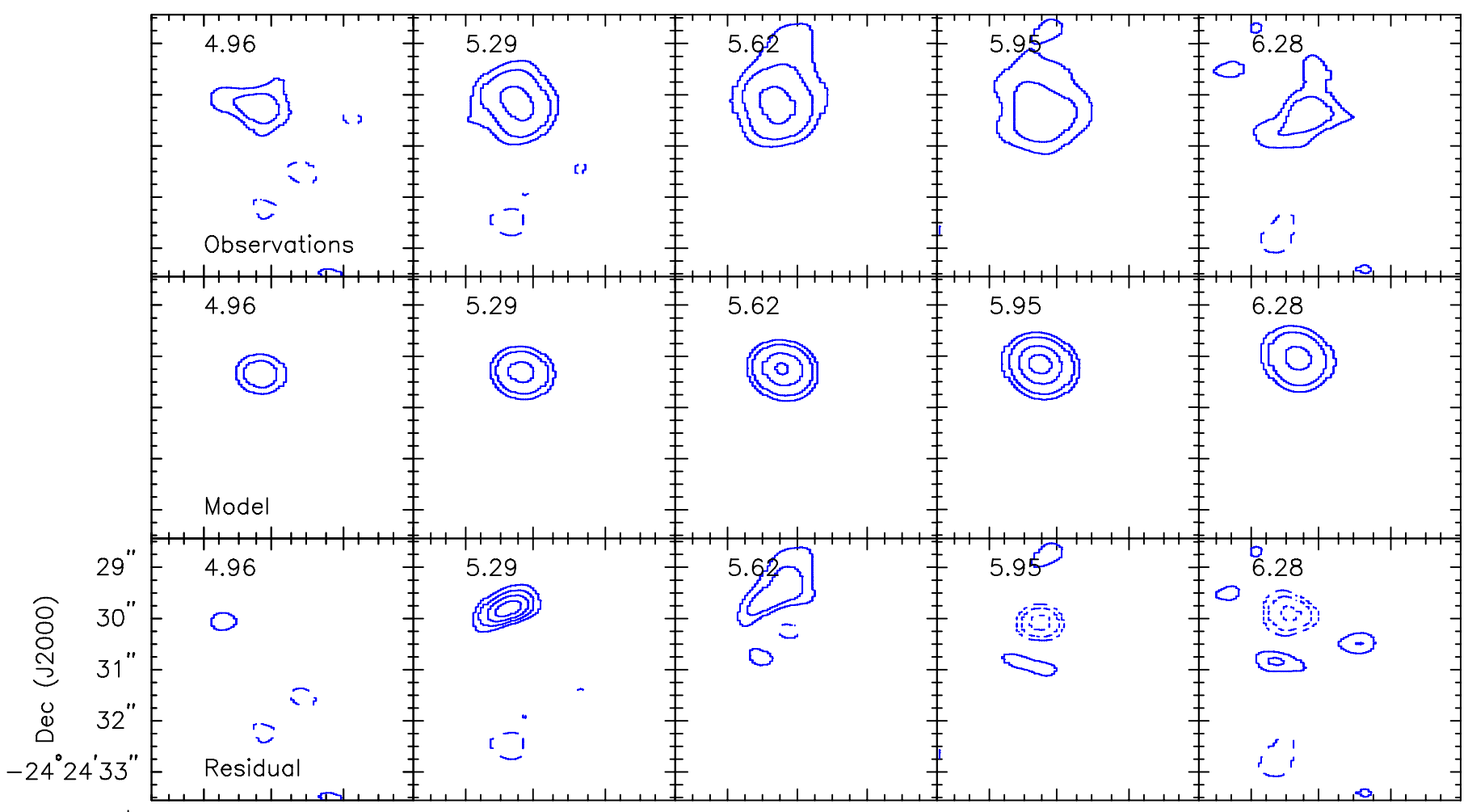

$16^{\mathrm{h}} 26^{\mathrm{m}} 26^{\mathrm{s}} .526^{\mathrm{s}} .426^{\mathrm{s}} \cdot 3 \quad 26^{\mathrm{s}} .2$

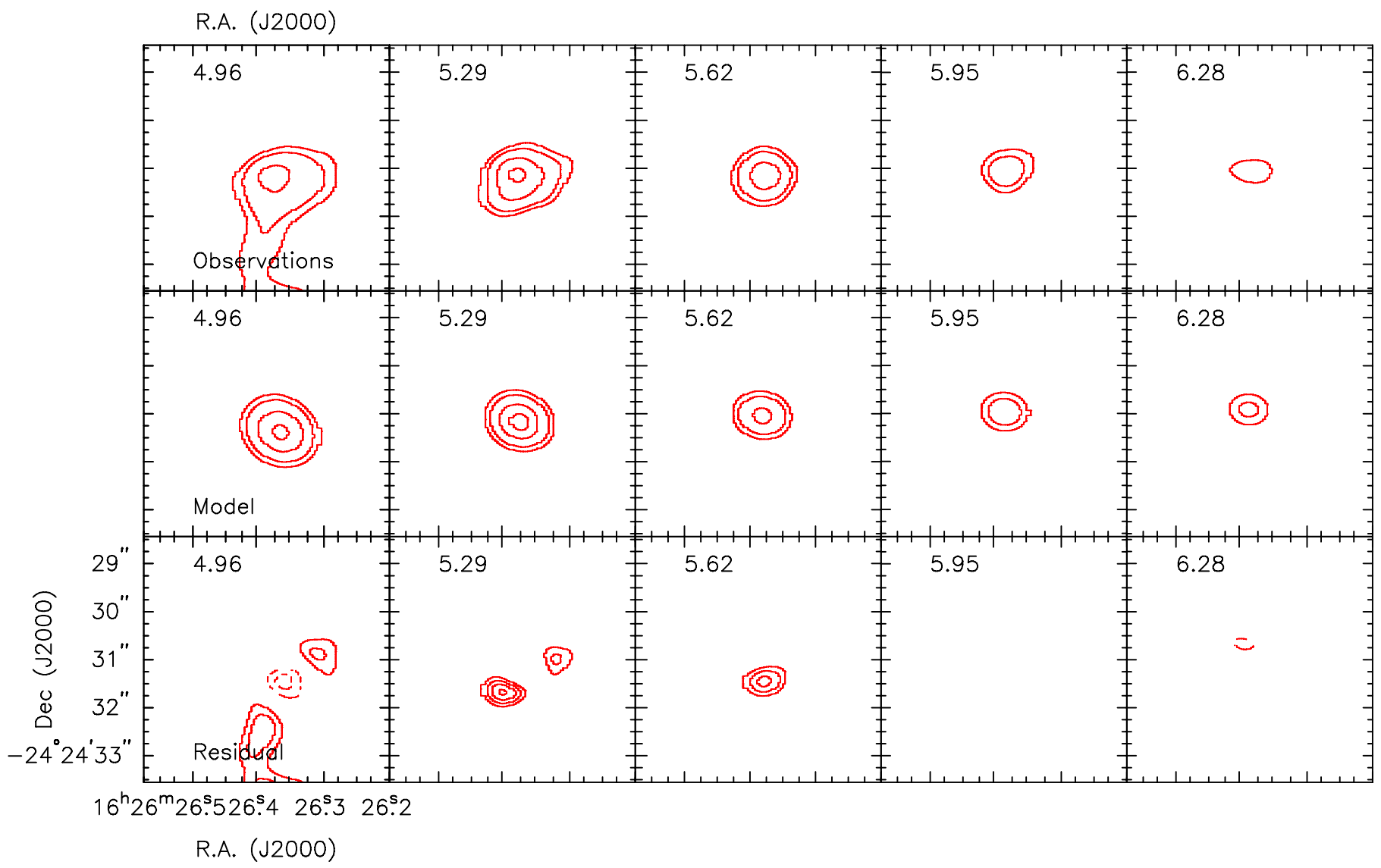

Fig. B.3. Same as Fig. B.1 but for the Conserved Angular Momentum disk model shown in Fig. 6. Note the large negative residuals in the blueshifted emission. 
N. M. Murillo et al.: A Keplerian disk around a Class 0 source: ALMA observations of VLA1623A

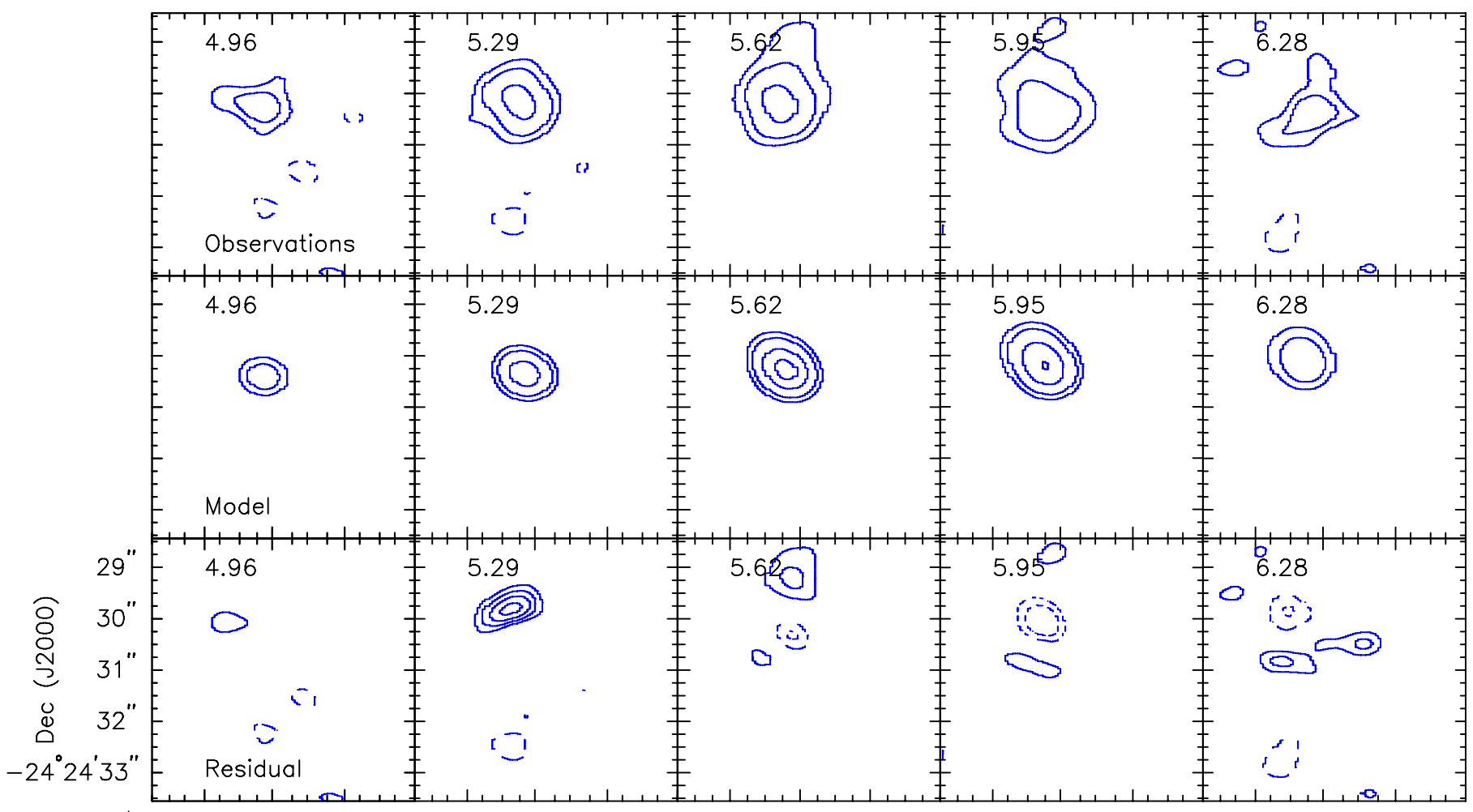

$16^{\mathrm{h}} 26^{\mathrm{m}} 26^{\mathrm{s}} .526^{\mathrm{s}} \cdot 426^{\mathrm{s}} \cdot 326^{\mathrm{s}} \cdot 2$

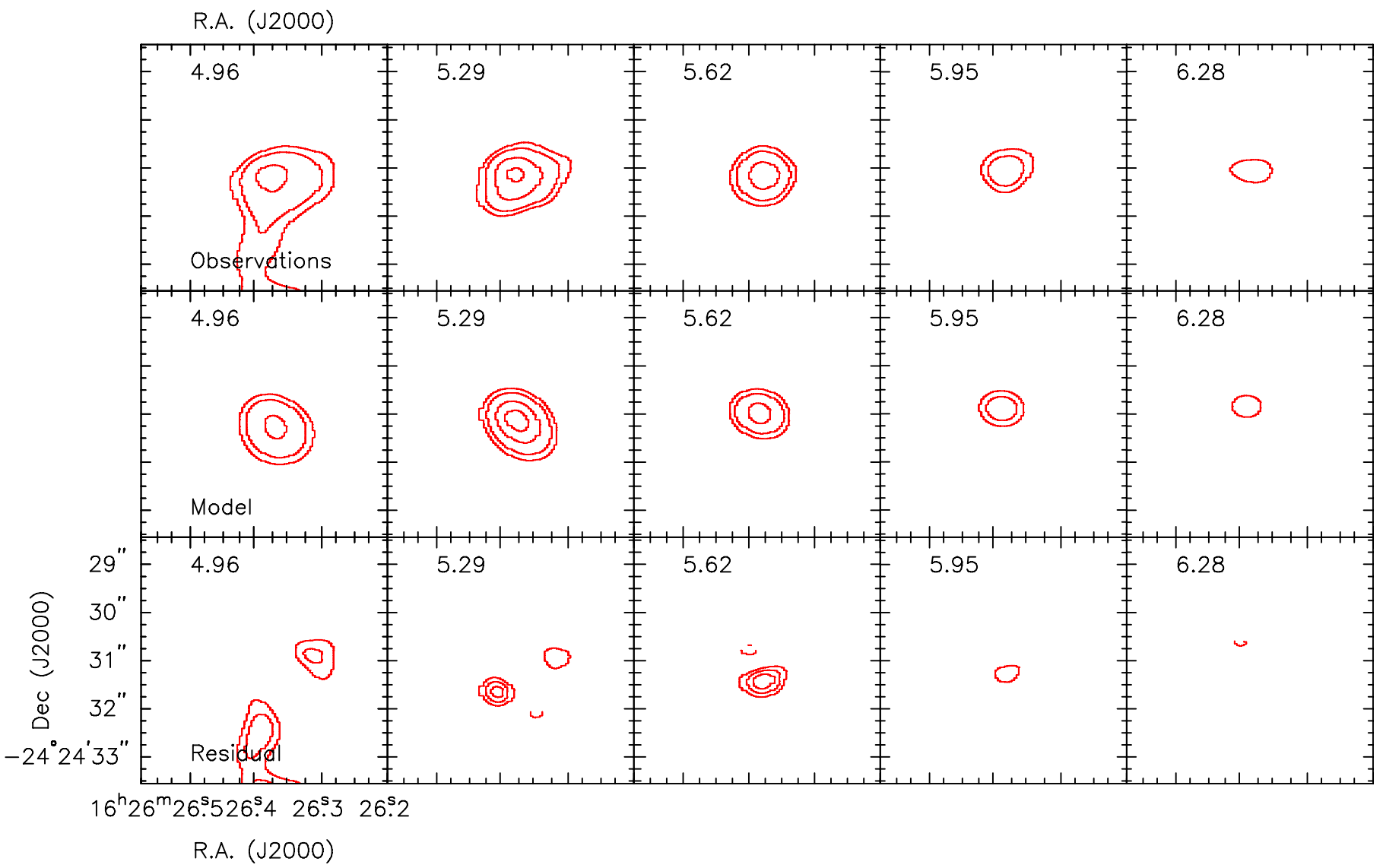

Fig. B.4. Same as Fig. B.1 but for the Infall plus Keplerian disk model shown in Fig. 6. 
A\&A 560, A103 (2013)

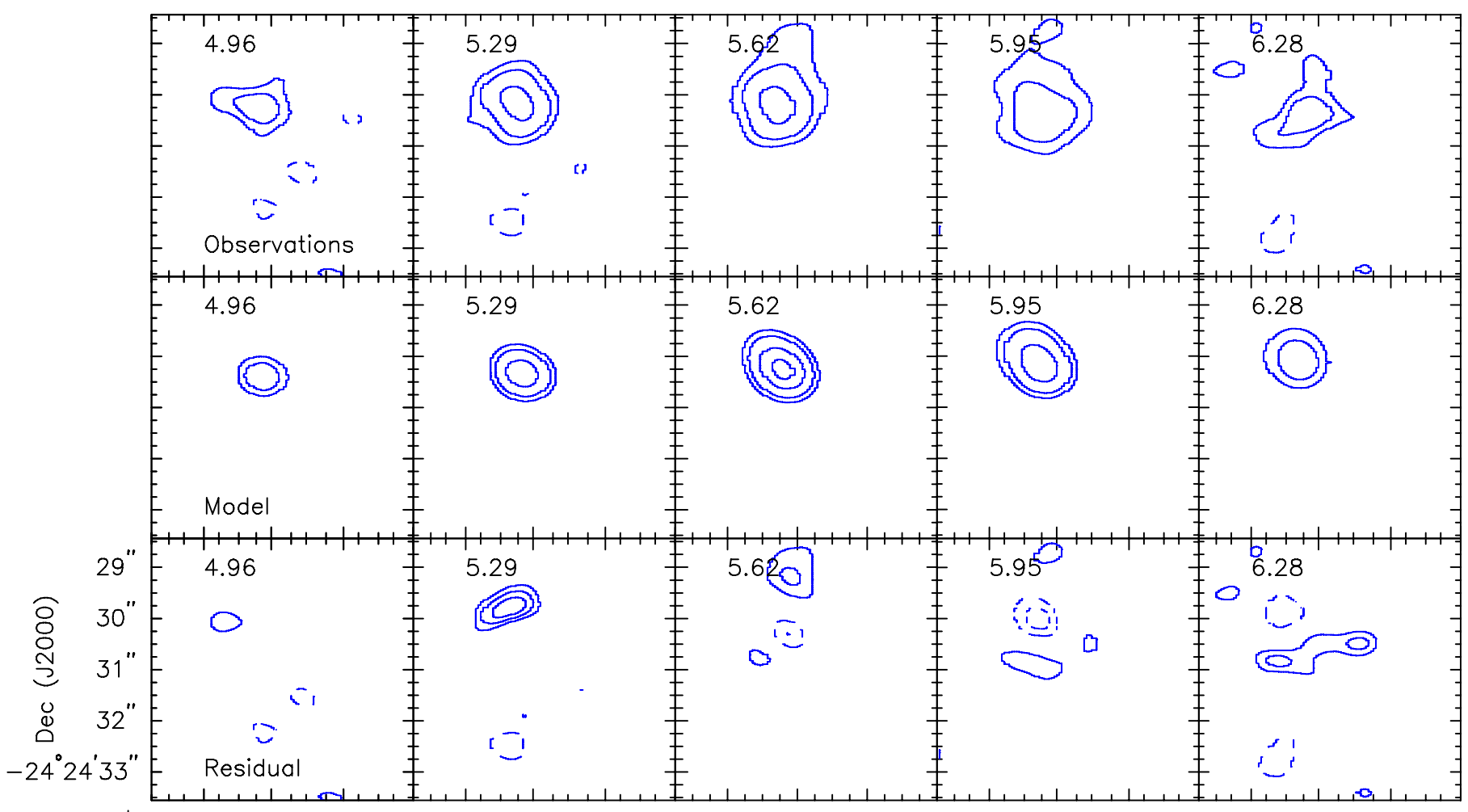

$16^{\mathrm{h}} 26^{\mathrm{m}} 26^{\mathrm{s}} .526^{\mathrm{s}} \cdot 426^{\mathrm{s}} \cdot 326^{\mathrm{s}} \cdot 2$

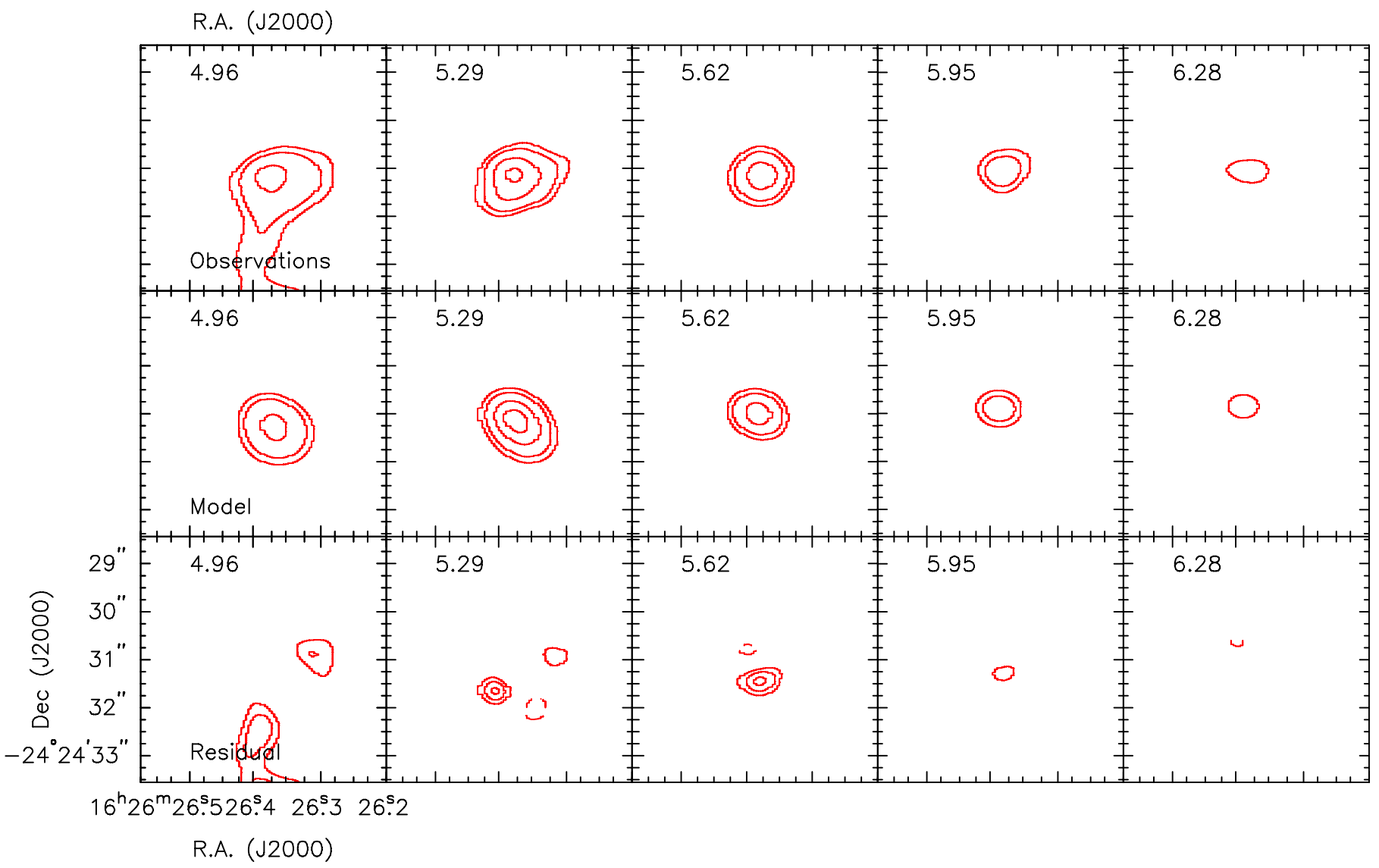

Fig. B.5. Same as Fig. B.1 but for the best fit pure Keplerian disk model shown in Fig. 6. 